\title{
An Analysis of the Origin of the Interaction Force between Electric Charges, including Justification of the $\ln r$ Term in the Completed Coulomb's Law, in HM16 Ether
}

\author{
Ioan Has ${ }^{1}$, Simona Miclaus ${ }^{1}$, Aurelian Has ${ }^{2}$ \\ ${ }^{1}$ Land Forces Academy "Nicolae Balcescu”, Sibiu, Romania \\ ${ }^{2}$ Independent Researcher, Rm. Valcea, Romania \\ Email: hasavo@yahoo.com,simo.miclaus@gmail.com, hasaurelian@yahoo.com
}

How to cite this paper: Has, I., Miclaus, S. and Has, A. (2019) An Analysis of the Origin of the Interaction Force between Electric Charges, including Justification of the $\ln r$ Term in the Completed Coulomb's Law, in HM16 Ether. Journal of Modern Physics, 10, 1090-1124.

https://doi.org/10.4236/jmp.2019.109071

Received: July 5, 2019

Accepted: August 3, 2019

Published: August 6, 2019

Copyright $\odot 2019$ by author(s) and Scientific Research Publishing Inc. This work is licensed under the Creative Commons Attribution International License (CC BY 4.0).

http://creativecommons.org/licenses/by/4.0/

\begin{abstract}
In this study, we demonstrate the correctness of our 2010 hypothesis regarding the need to complete Coulomb's $F_{C}$ law with the term $\ln r$, resulting in the completed $F_{C C}$ force. For this purpose, we consider the electrical interactions between charged microparticles (MPs), which develop as fundamental vibrations (FVs) in ether, producing the vibrational strains $\varepsilon$ and $\gamma$ and the resulting stresses $\sigma$ and $\tau$, as percussions of ether cells (ECs) upon the MP surface. The stresses $\sigma$ and $\tau$ produce a resultant force $F_{P}$ due to the percussions which constitute the real electric force $F_{C C}$ The spatial effect of ether on $F_{P}$ is demonstrated by an analytical method, considering the electrical interaction between MPs through various equidistant spatial paths $l_{i}$ of FVs, modelled on the basis of the Huygens principle for waves. For this issue, we utilized a numerical calculation, which could be generalized. But this spatial effect of the ether leads at a very slow decreasing of the $F_{P}$ forces ratio $r_{F}$ when doubling the distance $l$, in contrast to Coulomb's $F_{C}$ forces whose ratio $r_{F}$ decreases accentuate with doubling 1 . Accordingly, the necessity of including the term $\ln r$ in the $F_{C C}$ force, which is limited to 1.0 for doubling $l$, at long distances, was justified.
\end{abstract}

\section{Keywords}

Nature of Electric Charges, Constitution of Microparticles, Calculus of Electric Forces from Percussions Forces, HM16 Model of Ether with Fundamental Vibrations, Completed Coulomb Law Justification 


\section{Introduction}

The HM16 ether model, originally proposed by the present authors in 2016 [1], will be the starting point for the present analysis, which will provide new developments in the model in terms of the composition, and behaviour and effect of ether in nature and in physics.

We consider in our HM16 model that in nature, all microparticles (MPs) have either a positive or a negative electric charge, denoted $\mathrm{MP}^{+}$and $\mathrm{MP}^{-}$. However, in the general case in which the MP sign (+ or -) is not important, we will utilize the simple notation $\mathrm{MP}$ in the text, for both $\mathrm{MP}^{+}$and $\mathrm{MP}^{-}$. In addition, it is accepted in physics that at the macro scale, $\mathrm{MP}^{+}$and $\mathrm{MP}^{-}$are in parity, or even in pairs, and matter is neutral in nature.

To date, in physics, the nature of positive $+q$ and negative $-q$ electric charges has been neither defined nor explained. Furthermore, it has not been justified physically and materially.

In the following sections, we will initially present the modelling of the physical nature of the electric charge by highlighting new properties of the HM16 model of ether and of MPs, related to electric charges.

The paper brings in some novelties, such as the mechanical percussion nature of corrected electric forces $F_{C O}$ or the many simultaneous paths in ether of electric force $F_{C C}$ transmission from close to closer distances, in contrast to the classical Coulomb force $F_{C}$ transmitted at a distance.

The main new contribution of the paper is the analytical demonstration that the $F_{C C}$ forces decrease very slow with the distance $l$, thus justifying the new term $\ln r$, in contrast to the classical Coulomb force $F_{C}$ which decreases with distance.

But this spatial effect of the ether leads at a limit to 1.0 in the decrease of the $F_{P}$ forces ratio when doubling the distance 1 , in contrast to Coulomb's $F_{C}$ forces whose ratio decreases to 0.25 with doubling $l$.

Accordingly, the necessity of including the term $\ln r$ in the $F_{C C}$ force, which too is capped to 1.0 for doubling $l$, at long distances, was demonstrated.

In all the following graphical representations of space filled with ether, we will also indicate the indispensable reference frame (RF), usually as a Cartesian $O x y z$ frame, attached to a certain MP immovable in ether or to a certain cell of the ether (EC). Hence any $O x y z$ frame will be an absolute RF, referring to the ether.

\section{Origin of the Interaction Force between Microparticles with Electric Charges MP+ and MP- in the Case of the HM16 Ether Model}

Since we admit that the electric charge is intimately linked to MPs, the electric charge can be explained by the composition and functioning of MPs in the HM16 ether model.

In the following analysis, we will utilize the same abbreviations used in [1], which will be redefined in this section as follows.

HM16 is our ether model initiated in 2016, MP stands for microparticle, SMP 
for submicroparticle, ESMP for a especial submicroparticle, RF for the reference frame, EC for ether cells, EP for etheron particles, FV for fundamental vibrations in ether, $\mathrm{PV}$ for particle vibrations, $\mathrm{MB}$ for a material body, $F_{C C}$ for the corrected/completed Coulomb force, $F_{C}$ for the classical Coulomb electric force, $p_{i}$ for individual percussion forces, $f_{i}$ for continue force from percussions, $F_{P}$ for the resultant percussion force, $F_{N}$ for the classical Newtonian gravitational force, $F_{D C}$ for the force between two electrical dipoles, $r_{F}$ for $F_{s}$ forces ratio at doubled distances, $\varepsilon$ and $\gamma$ for linear and angular deformations/strains and $\sigma$ and $\tau$ for normal and tangential efforts/stresses;

An MP which is currently considered electrically neutral, such as a neutron, in fact comprises the MPs (or submicroparticles (SMPs)) $\mathrm{MP}^{+}$and $\mathrm{MP}^{-}$, with compensatory positive $(+q)$ and negative $(-q)$ electric charges, resulting in the common neutral bodies. In the following text, charge refers only to electric charge.

A first variant of the mode of operation of MPs considers the Type A HM16 ether model in terms of the crystalline disposition of etherons, as defined in [1], in which the specific vibrational/vortex model of an MP is considered as a basic phenomenon which transmits its vibrations to the surrounding crystalline network of etherons and accordingly, to other MPs (Figure 1).

A second variant of MPs' mode of operation is given by the Type B HM16 model of ether, in the form of a fluid as defined in [1], in which the flow/circulation of etheron particles (EPs) between different MPs occurs during the absorption or emission of ether particles or ether cells (ECs) by any MP. The $+q$ and $-q$ electric charges are given here by the debit of the fluid emitted or absorbed by specific MPs.

The Type A HM16 ether model is the main focus of the present analysis.

Of course, the detailed mechanisms developed between ether components and the forces acting between MPs, which will be presented and utilized in the next part of this paper, are only hypothesized, because until now it has not been possible to observe or directly measure them at their very small scale.

However, our HM16 ether model and its functioning is based on, and is in agreement with, actual existing experimental data from physics concerning the behaviour of matter and radiation at an observable scale.

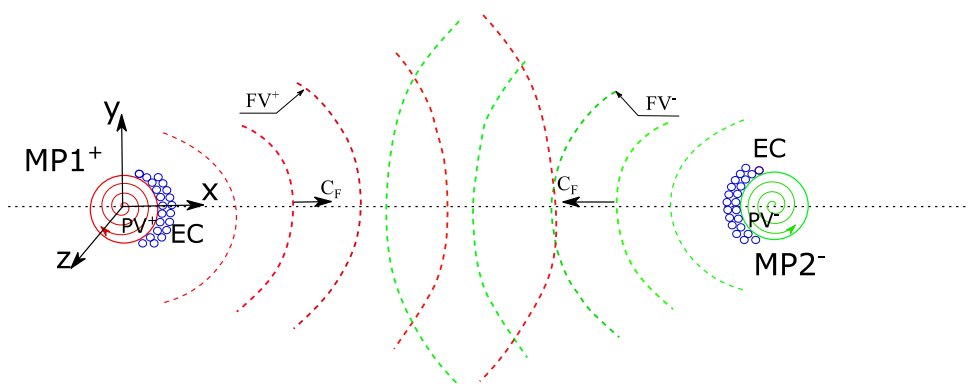

Figure 1. Interaction between $\mathrm{MP}^{+}$and $\mathrm{MP}^{-}$through the transmission of fundamental vibrations (FVs) in the Oxyz reference frame attached to the immovable MP1. 
Any such hypothesis will be confirmed in physics, when the results correspond to the real behaviour of matter and to the structure/composition of radiation at an observable scale.

Furthermore, as the purpose of this study, we will show that the results yielded by our HM16B model will be in accordance with the results of physical experiments. Accordingly, our initial hypothesis concerning the HM16 ether model will be confirmed.

It should be noted, as in [1], that the first-order components of HM16 ether, namely the ether particles themselves, are of two types: $\mathrm{EP} \alpha$ and $\mathrm{EP} \beta$. These are the $\alpha$ and $\beta$ etherons, which are grouped into the elemental cells (ECs) of ether, with at least four etherons (tetrahedrons) or as is more justified by volume symmetry, with eight etherons (cubes).

Ether is considered to be in its natural, undisturbed state, thus presenting no deformations/displacements but containing the fundamental vibrations (FVs) induced by all existing MPs, which are considered immobile/fixed in space at the starting moment of any analysis.

We acknowledge that the MPs in ether constitute physical support for the electric charges $+q$ and $-q$ and we refer to them as $\mathrm{MP}^{+}$and $\mathrm{MP}^{-}$respectively.

Firstly, in the HM16 ether model, any $\mathrm{MP}^{+}$or $\mathrm{MP}^{-}$contains a group of vibrating EPs or ECs exhibiting their own $\mathrm{PV}^{+}$or $\mathrm{PV}^{-}$vibrations, with their specific sign pattern (+/-) and with their own frequency $\omega$, where PV signifies an internal particle vibration (Figure 1).

Furthermore, the different actions of $\mathrm{PV}^{+}$and $\mathrm{PV}^{-}$produced by $\mathrm{MP}^{+}$and $\mathrm{MP}^{-}$ respectively, create corresponding $\mathrm{FVs}$ in the surrounding ether, i.e. $\mathrm{FV}^{+}$and $\mathrm{FV}^{-}$.

Any MP1 will produce its own FVs in ether, which travel away from the MP1 (Figure 1) and are eventually transmitted to all the other MPs in the ether from across the universe, according to the Huygens principle for vibrations.

We should state that, similarly to the claims made in section III of [1], the FVs of the HM16 ether comprise the linear specific (unitary) deformations $\varepsilon$ and the angular specific (unitary) distortions $\gamma$, of the crystalline spatial net of ECs. Both $\varepsilon$ and $\gamma$ are periodic or pulsatory deformations, with frequency $v$ corresponding to the pulsation $\omega$ of the MPs' rotations or oscillations. These specific deformations $\varepsilon$ and $\gamma$ of a body, are known in mechanics as strains.

In the present analysis, we will discuss only the plane problem of deformations or of the strains $\mathcal{E}$ and $\gamma$. Although in the general analysis of the HM16 ether model we are dealing with a spatial problem, in which the plane deformations $\varepsilon$ and $\gamma$ are replaced by the three-dimensional spatial tensors of deformations $\varepsilon$ and $\gamma$, this does not diminish the utility of studying the plane problem, which can then be generalized.

These vibrations in ether $\mathrm{FV}^{+}$and $\mathrm{FV}^{-}$will create the effect and mode of interaction between MPs, as the electrical forces, which are specific to $\mathrm{MP}^{+}$and different to $\mathrm{MP}^{-}$(Figure 2). 


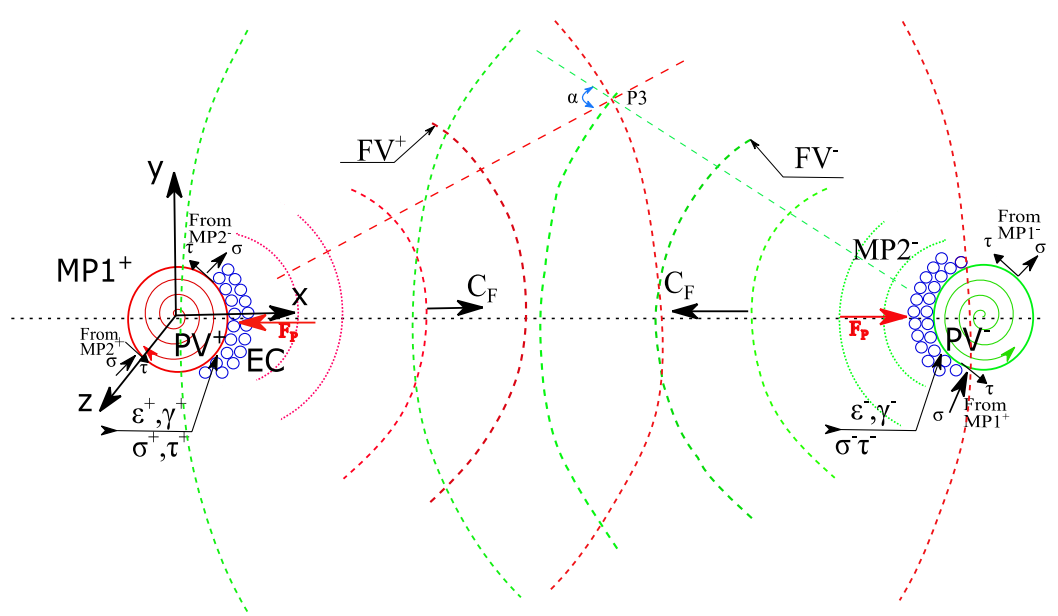

Figure 2. The emergence of the $F_{P}$ force between $\mathrm{MP}^{+}$and $\mathrm{MP}^{-}$created by the $\sigma$ and $\tau$ stresses, through the transmission of the FVs with the $\varepsilon$ and $\gamma$ strains, in the $O x y z$ reference frame attached to the immovable MP1.

This difference in the behaviour of $\mathrm{MP}^{+}$and $\mathrm{MP}^{-}$may, in principle, be manifested by the sense of the $\varepsilon$ deformation (elongation $+\mathcal{E}$ or compression $-\mathcal{E}$ ) and by the sense of the $\gamma$ rotation (clockwise $+\gamma$ or anticlockwise $-\gamma$ ), which are deformations produced in the ether's spatial net of ECs, under the effect of $\mathrm{FV}^{+}$ and $\mathrm{FV}^{-}$as created by $\mathrm{MP}^{+}$or $\mathrm{MP}^{-}$.

This specific behaviour of $\mathrm{MP}^{+}$or $\mathrm{MP}^{-}$is illustrated in Figure 3, which presents $\mathrm{MP}^{+}$and $\mathrm{MP}^{-}$disposed in opposition on a principal diagonal D1, i.e. in the two opposing quadrants of a Cartesian Oxyz reference system.

There, $\mathrm{MP}^{+}$manifests its own vibrations $\mathrm{PV}^{+}$, with a frequency $v$ (or pulsation $\omega$ ) consisting of strain couples $+\mathcal{E}$ and $+\gamma$ along $+\mathrm{D} 1$, and similarly $\mathrm{MP}^{-}$manifests its own $\mathrm{PV}^{-}$consisting of the strain couples $+\mathcal{E}$ and $-\gamma$ along $+\mathrm{D} 1$ (or couples $-\varepsilon$ and $+\gamma$ along D1) (Figure 3 ).

Then, MPs in contact with surrounding ECs will transmit and create FVs, via their own PVs.

The FVs in the ether are also of two types: $\mathrm{FV}^{+}$representing strain couples with the same sign $(+\varepsilon$ and $+\gamma$ or $-\varepsilon$ and $-\gamma)$ and $\mathrm{FV}^{-}$representing strain pairs with opposite sign $(+\varepsilon$ and $-\gamma$ or $-\varepsilon$ and $+\gamma$ ).

Accordingly, in the HM16 ether model, the specific properties of electric charges $+q$ and $-q$ are intrinsically attached to $\mathrm{MP}^{+}$and to $\mathrm{MP}^{-}$by their specific mode of vibration $\mathrm{PV}^{+}$or $\mathrm{PV}^{-}$manifested mechanically.

The detailed mechanics of PVs (as oscillations, vortexes or other kinds of movements) is not yet known, but it is not strictly necessary here.

Concerning the mechanism of the transmission of vibrations of the electrical force $F_{C C}$ between electrically charged MPs via the ether, we advance here a hypothesis regarding the manifestation and continuous action of percussion forces $p_{1}$ between the involved physical objects considered to be in direct contact (MPs, ECs), in which force transmissions are produced from close to closer distances (and not at a distance), as will be presented in the next section of this paper. 


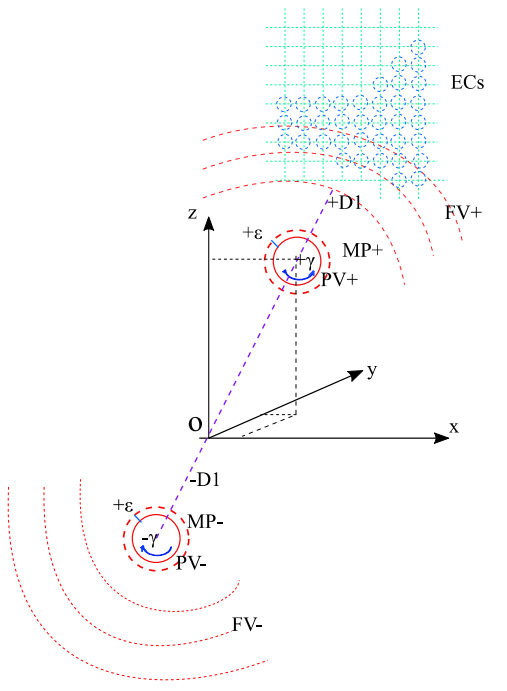

Figure 3. The manifestation of the charged $\mathrm{MP}^{+}$and $\mathrm{MP}^{-}$, creating the specific vibrations $\mathrm{FV}^{+}$and $\mathrm{FV}^{-}$in ECs, acting upon any MPs, creating the $\varepsilon$ and $\gamma$ strains and giving percussions $p_{p}$ in the $O x y z$ reference frame attached to an EC.

If an MP1 is present at a point in the ether and an MP2 is found in the area (Figure 2), the MP2 will be subjected over its entire external surface to a contact of FVs through the ECs, caused by MP1.

This contact action appears because the vibrating ECs of ether, around MP2, have direct contact with the MP2 surface and as such will create normal stresses $\sigma$ (two-way stresses, i.e. $-\sigma$ compression and $+\sigma$ stretching) and tangential stresses $\tau$ (with two senses $+\tau$ and $-\tau$ ) over the entire surface of the MP2 (Figure 2 ), with a resultant force $+f$ or $-f$ upon a unit area. Obviously, the phenomenon is similar for MP1 when the cause is an MP2.

In HM16, FVs caused by an $\mathrm{MP}^{+}$will create vectors (tensors in $3 \mathrm{D}$ ) of the stresses $\sigma$ and $\tau$ (or of the resultant force $f$ ) on the unit surface of an MP, disposed in the opposite directions to the $\sigma$ and $\tau$ stresses (or force $f$ ) caused on the same MP unit surface by an $\mathrm{MP}^{-}$.

Adding the two types of stress vectors (tensors) $\sigma$ and $\tau$ (or the resultant $f$ ) upon the whole surface of an MP will result in a $+F$ force of attraction or a $-F$ force of repulsion (Figure 2, Figure 3) between the two types of MPs, depending on whether they have identical or opposite electric charges $+q$ or $-q$.

Accordingly, in HM16, in the presence of $\mathrm{MP}^{+}$(Figure 2, Figure 3), the vectors of the strains $\varepsilon$ and $\gamma$ produced in the ether, as well as the resultant vectors of the stresses $\sigma$ and $\tau$ (or resultant $f$ ) acting on the $\mathrm{MP}^{-}$surface, will have opposite senses, resulting in an attraction force $+F$ on this $\mathrm{MP}^{-}$, while the same $+F$ force will be simultaneously applied to the $\mathrm{MP}^{+}$.

Regarding the resultant force caused by vibrational waves starting from two MPs of the same sign $\left(\mathrm{MP}^{+}\right.$and $\mathrm{MP}^{+}$or $\mathrm{MP}^{-}$and $\left.\mathrm{MP}^{-}\right)$, the vectors of the strains $\varepsilon$ and $\gamma$ and the vectors of the stresses $\sigma$ and $\tau$ (or resultant $f$ ) will have the same senses (Figure 2, Figure 3). In turn, there will be a repulsive force $-F$ on the 
second $\mathrm{MP}\left(\mathrm{MP}^{+} / \mathrm{MP}^{-}\right)$, while the same repulsive force $-F$ will appear on the first MP at the same time.

Consequently, the difference in the behaviour of interactions between $\mathrm{MP}^{-}$ and $\mathrm{MP}^{+}$is manifested according to the above presentation.

However, it is possible that between MPs of the same sign, attraction forces arise (and between the MPs of opposite signs, repulsion forces appear) according to the completed law of $F_{C C}$ force [2]. For these forces, there will be different formulas, that depend on the distance $I_{i}$ between the MPs or between the material bodies (MBs).

However, these interaction forces, having the opposite sense to those usually currently considered, will not change the physical behaviour of the MPs, which possess a property equivalent to the actual sign of the electric charge.

The above presentation can be considered to be the mechanism of the appearance and manifestation of electric charges $+q$ and $-q$. They are, in fact, intrinsic properties of MPs and of the ether.

$\mathrm{MPs}^{+}$and $\mathrm{MPs}^{-}$produce in ether some specific $\mathrm{FV}^{+}$or $\mathrm{FV}^{-}$which acts upon other $\mathrm{MPs}^{-}$and MPs ${ }^{+}$. Depending on these FVs, the interaction force $F_{C C}$ results between MPs.

Today, in physics, the Coulomb force $F_{C}$ is considered to be a manifestation of interaction at distance between the so-called electric charges $+q$ and $-q$. However, these charges do not exist as physical entities, only as manifestations of specific vibrations of MPs and of the HM16 ether model (Figure 3).

Note that the sense of the $\mathrm{F}_{\mathrm{DC}}$ force between two electric dipoles will always be attraction [2], resulting in a gravitational pull exerted by the $F_{D C}$ via the corrected $F_{C C}$ force given in Euation (21) in [3].

\section{The Occurrence Mode of the Interaction Force between Charged Microparticles in the Case of Type A HM16 Ether}

To the best of our knowledge, in no classical analysis of electricity [4] [5] [6] [7]

[8] is there any discussion of the possibility that the Coulomb forces $F_{C}$ could, in fact, be the result of the unitary forces $\sigma$ or $\tau$, which might act on the surface of MPs due to $\varepsilon$ or $\gamma$ deformations of the ether.

We note that in the classical approach, considering the Coulomb electric interaction between two MBs or MPs endowed with electric charges $\pm q$, only the existence of concentrated $F_{C}$ forces of the Coulomb type are acknowledged.

It is also classically understood that $F_{C}$ forces appear and act at a distance instantly and mutually between MB1 and MB2, with the sense of the action provided by the electric charge signs $\pm q$. The $F_{C}$ force is considered to be a linear, one-directional vector, which is transmitted directly on a straight line between $\mathrm{MB} 1$ and MB2.

We observe that, similar to the case of the electric force, in the case of Newtonian gravitational interaction, the gravitational force $F_{N}$ is classically considered to act between two MBs. It is acknowledged that the force $F_{N}$ is also a focused 
force, the size of which is a function of the masses $m$ of the bodies and of the distance $1 / r^{2}$ [7] [8].

However, in our analysis of the presence of HM16 ether, we consider that the cause of $F_{C}$ forces is the appearance of the unitary stresses $\sigma$ and $\tau$ acting on the entire surface of an MP2 (Figure 2). Furthermore, the $\sigma$ and $\tau$ stresses do not occur instantly at a distance but are the result of the specific $\varepsilon$ and $\gamma$ deformations of the ECs around the MP2. In addition, $\varepsilon, \gamma$ strains are produced by FVs from ether, caused by the PVs vibrating permanently inside the corresponding MP1, whose strains $\varepsilon$ and $\gamma$ are permanently transmitted by the MP1 directly to the ether as FVs.

In this case, in the presence of HM16 FVs in the ether, they neither occur nor are transmitted between MP1 and MP2 in the form of a single straight vector of the $F_{C}$ type. In fact, these fundamental waves/FVs are transmitted in the form of a spatial phenomenon, which is present around any MP in the form of spatial vibrations/waves advancing from MP1 in all directions in space at a speed $c_{f}$ (Figure 2). The FVs are transmitted through the material structure as ECs of the HM16 ether, starting from any existing $\mathrm{MP}_{\mathrm{i}}$ and continuing according to the Huygens principle into the whole ether present in the whole of space, which in this case is considered to be Euclidean space.

Moreover, the classical transmission of $F_{C}$ as a linear vector from MP1 directly to the destination (MP2) cannot occur, because it is highly likely that the $F_{C}$ straight line will encounter another MP, or even several MPs, existing in any MBs. Furthermore, such a classical type straightforward $F_{C}$ force in the form of a straight line (linear vector) cannot bypass an obstacle, or even an MP. As a result of bypassing an $\mathrm{MB}$, the $F_{C}$ force can no longer be considered to be a linear vector or a straight line.

Due to the above situation, the spatial transmission of $F_{C C}$ in the form of spatial FVs, in terms of ether volume, prompts an additional argument.

In fact, the $\varepsilon$ and $\gamma$ deformations of the ether acting on MP2 are caused by the corresponding MP1 itself. Furthermore, MP1 is in an area of the ether where a quantity of energy $(\Delta E)$ has accumulated, which is manifested in the production of its own PVs as vortexes/oscillations in the EC group in the volume of the MP1 (Figures 1-3).

These PV vibrations in the EC group constituting the MP1 may in fact be periodic deformations (or even rotations/whirlpools) inside these ECs from MP1, of type $\varepsilon_{p}$ and $\gamma_{p}$ and with frequency $\omega$ (Figures 1-3). They are similar to the two types of specific deformations in compact MBs, known in mechanics as $\varepsilon_{m}$ and $\gamma_{m}$. However, $\varepsilon_{p}$ and $\gamma_{p}$ do not transmit energy like $\varepsilon_{m}$ and $\gamma_{m}$, owing to the ether's special (ideal) properties.

Meanwhile, MP1, by being in intimate contact with ether, precisely conveys its vibrations to it as $\varepsilon_{p}$ and $\gamma_{p}$ deformations, which will create periodic $\varepsilon$ and $\gamma$ deformations in the ECs of the adjacent ether (Figure 2, Figure 3). The $\varepsilon$ and $\gamma$ deformations of ether will have an amplitude that may be different from that of 
$\varepsilon_{p}$ and $\gamma_{p}$ from the MP, but they will have their own preserved frequency $\omega$ across the entire network of the surrounding ECs.

It follows that these $\varepsilon$ and $\gamma$ deformations in the whole EC network are continuously present over time, as long as there exists an MP1 at their origin and no energy consumption/transmission is involved. In addition, these $\varepsilon$ and $\gamma$ deformations in ether can only be transmitted from near to near in the whole three-dimensional ether network, with a fundamental speed $c_{f}$ given by ether's physical properties. These properties are currently unknown, but we only can suppose they are the properties of an ideal body having an extremely high modulus $\mathrm{E}$ and an extremely low density $\rho$, consequently resulting in a wave speed $c_{\mathrm{f}}$ that is extremely high, in our estimate.

At the same time, it is likely that in the fixed ether cell/particle (EC/EP) network, $\varepsilon$ and $\gamma$ deformations can only be produced in quantum terms, by altering the positions of ECs/EPs in the ether only in jumps of $\Delta x, \Delta y$ and $\Delta z$. These jumps can only take place between fixed EC/EP positions in the primary ether network (Figure 2, Figure 3), or possibly between intermediate positions in the second-order ether network [1].

Such a primary network is considered to be fixed, with stable EC positions in space, including in the fixed $\mathrm{Oxyz}$ reference frame (Figure 2, Figure 3), and permanent in time if disturbing factors do not occur.

Now, we consider such an etheric network with a given point P2, situated upon an MP2 surface (Figure 2). At time $t$ at P2, the FVs arise from one, two or more sources (MP1a, MP1b, MP1c, etc.), located in the area in approximately the same direction (Figure 2). Then, local vibrational deformations $\varepsilon$ and $\gamma(\omega)$ of the ether will appear at $\mathrm{P} 2$. At the point $\mathrm{P} 2$ at which these vibrations arrive on the MP2 surface, we can say that the FVs have reached their end point or terminus point. Here, the interesting, useful deformations $\varepsilon$ and $\gamma(\omega)$ can only be those in the last layer of ECs in direct contact with the surface of the MP2, caused by all MP1s existing in the considered direction (Figure 2).

The P2 terminus point of the FVs' vibration paths can only be located at the point of contact of FV1 with an MP2 surface. For the MP2, the effect of the $\varepsilon$ and $\gamma(\omega)$ deformations in the surrounding ether will be manifested by the MP2's own EC deformations, i.e., $\varepsilon_{p}$ and $\gamma_{p}$. These deformations imply a modification of the stress distribution $\sigma, \tau$ on the surface of the MP2, resulting in the $F_{P}$ type of interaction force described in Section 4 , as a result of these stresses. Note that this $F_{p}$ force is the effect of FV1 through $\sigma$ and $\tau$, appearing on the entire outer surface of the MP2.

However, if the point P3 is located anywhere in the ether network in an area in which there is no MP (Figure 2), the path of FV1 will not have P3 as an end point, and so the FV1 waves will continue beyond P3. Beyond P3, there will be a continuation of the FV1 vibration transmission and of the $\varepsilon$ and $\gamma(\omega)$ ether deformation. Accordingly, at the $\mathrm{P} 3$ point, there is no longer an end-point effect of the deformation, as in the case of $\mathrm{P} 2$. 
Meanwhile, at the $\mathrm{P} 3$ point, which is not a terminus point, the continuation of the waveform path of FVs and the $\varepsilon$ and $\gamma(\omega)$ deformations of ether can be pursued according to the Huygens principle, which also applies to ether, as shown in Section 5.

This is because the ether constitutes a special, albeit material, structure, present in all space, so that anywhere there is a P3 free point, where an FV1 arrives, the Huygens principle of waves can be applied [7] [8]. According to the Huygens principle, the point $\mathrm{P} 3$ becomes a source of a secondary vibration FV1' of the ether, which has the same nature as FV1 but moves only in the forward direction.

However, if at the point $\mathrm{P} 3$ a second vibration FV2 arrives concomitantly with FV1, the travel direction of which makes an angle $\alpha$ with the direction of FV1 (Figure 2), it is logical that in P3, a certain effect or phenomenon of special interference of the Huygens type appears, in which both FV1 and FV2 will have an effect upon the ether, as will be shown in Section 4.

\section{Mechanism of the Occurrence and Calculation of the $F_{P}$ Force between the Considered Electrically Charged Microparticles, by a Real Interaction Force as a Result of Ether Percussions}

The FVs produced by all other MPs in the ether of the universe, including those of MP1 (Figures 1-3), act upon MP2.

Any of these FVs must be considered to act on the point P2 on the surface $S$ of MP2, via the strains $\varepsilon(\omega)$ and $\gamma(\omega)$, which create the stresses $\sigma$ and $\tau$ (Figure 2, Figure 3). However, it must be stated that the principle of the superposition of effects is valid, meaning that we can separately analyse the effects of MP1 (or any $\mathrm{MP}_{\mathrm{i}}$ ) upon MP2.

We can consider the plane problem further, even though the stress phenomenon occurs in space (Figure 4).

In fact, on the entire outer surface $S$ of MP2, there will be variations in stresses $\sigma$ and $\tau$, due to the differences in strains $\varepsilon(\omega)$ and $\gamma(\omega)$, which are precisely created on the surface $S$ of MP2 by the action of the FVs of the ether produced by MP1.

We can consider the action of any FV waves on PM2, by exerting small percussions $p_{i}$ with the FV frequency(pulsation) $v(\omega)$ (Figure 4). The percussions $p_{i}$ are the results of the variations in the stresses $\sigma$ and $\tau$ produced by any vibrating ECs, due to the FVs of the ether, consisting of the deformations $\varepsilon(\omega)$ and $\gamma(\omega)$ with frequency/pulsation $v / \omega$ of the FVs.

However, we consider that the small percussions $p_{i}$ of an EC, act with the frequency $v$ of the FVs (Figure 4). If the frequency of an FV is $v$, the EC and the produced $p_{i}$ will act $v$ times per second, and so the percussions $p_{p}$ which are discrete in time, will result in a continuous equivalent force $f_{i}$ of an EC, given by the equation 


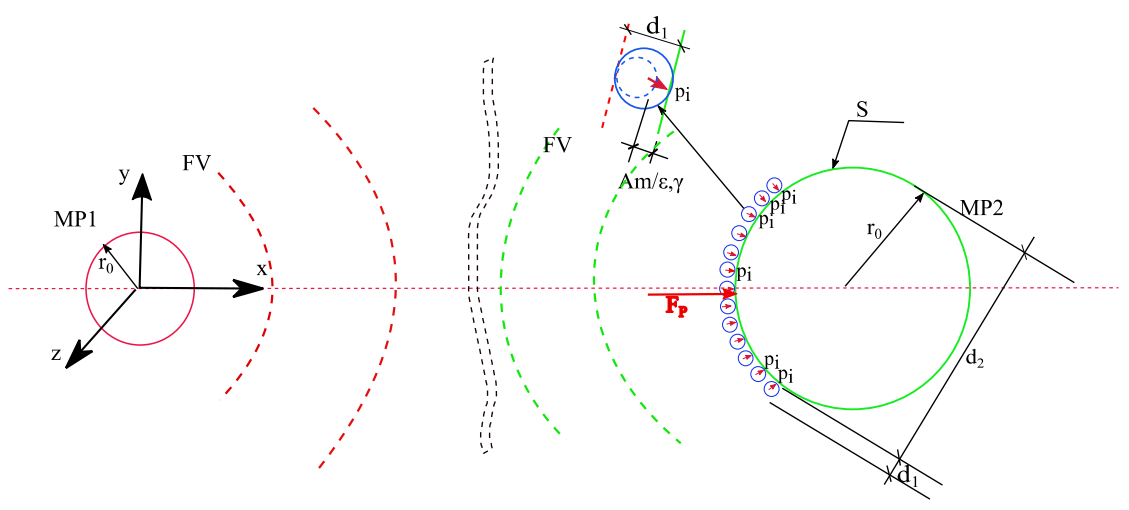

Figure 4. The mechanism of production of the percussions $p_{i}$ created by FV from MP1 to MP2, in the Oxyz reference frame attached to the immovable MP1.

$$
p_{i}=\frac{1}{v} f_{i}
$$

Physically, the magnitude of the percussion force $p_{i}$ will be given by the square of the amplitude $\left(A_{m}\right)^{2}$ of the displacement of the EC of the ether, at the time of the EC collision with MP2 (Figure 4). This is similar to the vibration energy of a harmonic oscillator. The magnitude of $p_{i}$ will also depend on the distance $I$ between MP1 and MP2, with a physically justifiable variation, along with the inverse of the square of the distance $P$, as in Coulomb's $F_{C}$ force Equation (1) in [1].

$$
p_{i}=k_{e} \frac{\left(A_{m}\right)^{2}}{l^{2}}
$$

In Equation (1), $k_{e}$ is an elastic coefficient of the type of Hooke's modulus $E$ from the field of mechanics:

$$
\sigma=E \varepsilon \text { or } E=\sigma / \varepsilon
$$

showing the proportionality between $p_{i}$ and the amplitude $A_{m}$. It follows that the measurement unit (in SI) of $k_{e}$ will be

$$
\left[k_{e}\right]=\frac{\left[p_{i}\right]\left[l^{2}\right]}{\left[A_{m}\right]^{2}}=\frac{\mathrm{N} \cdot \mathrm{s} \cdot \mathrm{m}^{2}}{\mathrm{~m}^{2}}=\mathrm{N} \cdot \mathrm{s}
$$

It follows that the measurement unit (in SI) of E from Equation (2) will be:

$$
[E]=\frac{[\sigma]}{[\varepsilon]}=\frac{\mathrm{N} / \mathrm{m}^{2}}{-}=\mathrm{N} / \mathrm{m}^{2}
$$

The unitary force $f_{i}$ given by an EC, which is considered to be a continuous force in time resulting from the percussions $p_{i}$ from an EC upon a MP, will be given by introducing (1) into (0), resulting in:

$$
f_{i}=v p_{i}=v k_{e} \frac{\left(A_{m}\right)^{2}}{l^{2}}
$$

We will make an approximate assessment of the force $f_{i}$ by adopting a possible 
value for the elastic constant $k_{e}$ from Equation (1). We do this by utilizing its similarity to the value of the elastic coefficient $E$ in Hooke's law for a solid crystalline body (closest to the behaviour of the Type $A$ ether in [1]).

For steel, we have the following value from mechanics.

$$
E \approx 2 \times 10^{11} \mathrm{~N} / \mathrm{m}^{2}
$$

Now, it follows that in Equation (2c) the expression with the same measurement unit (in SI) as $E$ in (2b) will be

$$
\left[\frac{v k_{e}}{l^{2}}\right]=\frac{\left[f_{i}\right]}{\left[\left(A_{m}\right)^{2}\right]}=\frac{\mathrm{N}}{\mathrm{m}^{2}}=\left[E^{\prime}\right]
$$

From Equations (2a) and (3) we find the equivalence of terms.

$$
k_{e}=\frac{E^{\prime} l^{2}}{v}
$$

We obtain a value of the ether modulus $E^{\prime}$ of about three orders of magnitude greater than that for steel from Equation (2d):

$$
E^{\prime}=10^{3} \times E=10^{3} \times 2 \times 10^{11} \mathrm{~N} / \mathrm{m}^{2} \approx 2 \times 10^{14} \mathrm{~N} / \mathrm{m}^{2}
$$

1). The case of astronomical distances. Now, we substitute in (3a) an astronomical distance $l=10^{7} \mathrm{~m}$, and a frequency for the FVs approximately the same as for $\gamma$ rays, i.e. $v=10^{22} \mathrm{~Hz}$, obtaining:

$$
k_{e}=\frac{2 \times 10^{14}\left(10^{7}\right)^{2}}{10^{22}}=2 \times 10^{6} \mathrm{~N} \cdot \mathrm{s}
$$

Here, we must consider the spatial problem of MP1 and MP2, with $f_{i}$ representing the modulus of a spatial vector $\overline{f_{i}}$, oriented normally at the sphere surface of MP2 at any point.

The resultant force $F_{P}$ given by the percussions produced by all the ECs in contact with MP2, with a total number of $n$ percussions acting permanently in time on the sphere surface $S$ of MP2 (Figure 4), will be the resultant sum of vectors $\bar{f}_{i}$ oriented normally on the entire surface $S$ of MP2.

Let us calculate approximately the number $n$ of ECs, for a diameter $d_{1}$, acting from the direction of MP1 on MP2 with diameter $d_{2}$ (Figure 4). We consider that the relevant area for the number $n$ of ECs in contact with MP2, is that of the spheres of MP2 and that of the large circle of the EC. This can be calculated as the ratio of the spherical surface $S$ of MP2 in contact with ECs and the area $s$ of the large circle around the EC sphere.

$$
n=\frac{S_{\mathrm{MP} 2}}{s_{\mathrm{CE}}}=\frac{\pi d_{2}^{2}}{1 / 4 \pi d_{1}^{2}}=4 \frac{d_{2}^{2}}{d_{1}^{2}}
$$

The ECs are located with distances between them of approximately $2 d_{1}$. We consider that the diameter $d_{1}$ of an EC is $d_{1}=b_{e}=10^{-27} \mathrm{~m}$, according to [1]. We consider that the diameter of an electron/proton MP is $d_{2}=10^{-15} \mathrm{~m}$. Entering these values into (4) results in the following number (pcs) of ECs in contact. 


$$
n=4 \frac{\left(10^{-15}\right)^{2}}{\left(2 \times 10^{-27}\right)^{2}}=4 \frac{10^{-30}}{4 \times 10^{-54}}=10^{24} \mathrm{pcs}
$$

We will consider that the FV amplitude $A_{m}$ is of the order of $10^{-3} d_{1}$, similar to the working strain $\varepsilon$ in the mechanics of solids.

The effects of percussions $p_{i}$ (and of $f_{i}$ ) acting at $360^{\circ}$ will be given by their projections on the Ox axis $f_{i x}$, which gives a mean projection angle of $45^{\circ}$, resulting in

$$
f_{i x}=f_{i} \cos 45^{\circ}=f_{i} \cdot 0.71
$$

However, $f_{i x}$ acts in two senses on the Ox axis, giving a distribution of approximately $+50 \%$ and $-50 \%$ in the two senses. The forces $+f_{i x}$ are given by the distance $I_{0}$ while the forces $-f_{i x}$ are given by the distance $\left(I_{0}+d_{1}\right)$. We can obtain from Equations (4b) and (2c):

$$
\begin{gathered}
f_{i 0}=\frac{0.71 \mathrm{vg}}{l^{2}} \text { with } g=k_{e}\left(A_{m}\right)^{2} \\
g=2 \times 10^{6}\left(10^{-3} \times 10^{-27}\right)^{2}=2 \times 10^{-54} \mathrm{~N} \cdot \mathrm{s} \cdot \mathrm{m}^{2}
\end{gathered}
$$

Using an astronomical distance $l_{0}=10^{7} \mathrm{~m}$ we can obtain from Equations (4b), (4c) and (4d):

$$
\begin{gathered}
\frac{1}{0.71} f_{i 0}=2 \times \frac{10^{22} \times 10^{-54}}{\left(10^{7}\right)^{2}}=2 \times \frac{10^{-32}}{\left(10^{7}\right)^{2}} \mathrm{~N} \\
\frac{1}{0.71} f_{i 1}=2 \times \frac{10^{22} \times 10^{-54}}{\left(10^{7}+10^{-15}\right)^{2}}=2 \times \frac{10^{-32}}{\left(10^{7}+10^{-15}\right)^{2}} \mathrm{~N}
\end{gathered}
$$

Hence the net force is given by the difference of the forces from Equations (4e) and $(4 \mathrm{f})$ :

$$
\begin{gathered}
\Delta f_{i}=f_{i 1}-f_{i 0}=0.71 \times 2 \times 10^{-32}\left[\frac{1}{\left(10^{7}+10^{-15}\right)^{2}}-\frac{1}{\left(10^{7}\right)^{2}}\right] \\
\Delta f_{i}=f_{i 1}-f_{i 0}=0.71 \times 2 \times 10^{-32} \frac{\left(10^{7}\right)^{2}-\left(10^{7}+10^{-15}\right)^{2}}{\left(10^{7}\right)^{2}\left(10^{7}+10^{-15}\right)^{2}} \\
=0.71 \times 2 \times 10^{-32} \frac{10^{14}-10^{14}-2 \times 10^{7} \times 10^{-15}-10^{-30}}{10^{14}\left(10^{14}+2 \times 10^{7} \times 10^{-15}+10^{-30}\right)} \\
\Delta f_{i}=f_{i 1}-f_{i 0} \approx 0.71 \times 2 \times 10^{-32} \times \frac{-2 \times 10^{7} \times 10^{-15}}{10^{14} \times 10^{14}} \\
=-0.71 \times 2 \times 10^{-32} \times \frac{2 \times 10^{-8}}{10^{28}}=-2.84 \times 10^{-68} \mathrm{~N}
\end{gathered}
$$

The total force of interaction $F_{p}$ upon an MP, given by the net percussions $\Delta f_{i}$ of all the ECs around it, can be calculated from Equations (4a) and (5b).

$$
F_{P}=n \times \Delta f_{i}=-10^{24} \times 2.84 \times 10^{-68}=-2.84 \times 10^{-44} \mathrm{~N}
$$


For comparison, we will calculate the classical Coulomb force $F_{O}$ using Equation (1) of [1]. In a situation in which MP2 has a charge $+e$ and MP1 has a charge $-e$, located at a distance of $I=10^{7} \mathrm{~m}$, this results in:

$$
F_{c}=-\frac{(1 e)^{2}}{4 \pi \times 8.85 \times 10^{-12} \times l^{2}}=-\frac{\left(1.6 \times 10^{-19}\right)^{2}}{4 \pi \times 8.85 \times 10^{-12} \times\left(10^{7}\right)^{2}}=-2.30 \times 10^{-42} \mathrm{~N}
$$

It is noted that in Equations (6) and (6a), the two forces $F_{P}$ and $F_{C}$ were obtained with values that were not equal but close, with $F_{P}<F_{C}$

This can also be seen by considering that the value of $F_{P}$ obtained in (6) is the force resulting from only one electric interaction path $l_{i}$ out of multiple interaction paths and multiple corresponding forces $F_{P P}$ as will be seen in Section 5 .

The difference is also due to the approximations of the physical parameters of ether and of the simplified forces $f_{p}$ made in the above numerical calculation.

2). The case of a laboratory distance. Now, we substitute in Equation (3a) a laboratory distance $l=10^{-1} \mathrm{~m}$ and the same frequency for the $\mathrm{FVs}$, and we obtain

$$
k_{e}=\frac{2 \times 10^{14}\left(10^{-1}\right)^{2}}{10^{22}}=2 \times 10^{-10} \mathrm{~N} \cdot \mathrm{s}
$$

From Equations (4c) and (7) we obtain:

$$
g=2 \times 10^{-10}\left(10^{-3} \times 10^{-27}\right)^{2}=2 \times 10^{-70} \mathrm{~N} \cdot \mathrm{s} \cdot \mathrm{m}^{2}
$$

With a laboratory distance $l_{0}=10^{-1} \mathrm{~m}$ we can obtain from Equations (4e), (4f) and $(7 b)$ :

$$
\begin{array}{r}
\frac{1}{0.71} f_{i 0}=2 \times \frac{10^{22} \times 10^{-70}}{\left(10^{-1}\right)^{2}}=2 \times \frac{10^{-48}}{\left(10^{-1}\right)^{2}} \mathrm{~N} \\
\frac{1}{0.71} f_{i 1}=2 \times \frac{10^{22} \times 10^{-70}}{\left(10^{-1}+10^{-15}\right)^{2}}=2 \times \frac{10^{-48}}{\left(10^{-1}+10^{-15}\right)^{2}} \mathrm{~N}
\end{array}
$$

Hence, the net force $\Delta f_{i}$ given by the difference of the forces $f_{i}$ above, will be:

$$
\begin{gathered}
\Delta f_{i}=f_{i 1}-f_{i 0}=0.71 \times 2 \times 10^{-48}\left[\frac{1}{\left(10^{-1}+10^{-15}\right)^{2}}-\frac{1}{\left(10^{-1}\right)^{2}}\right] \\
\Delta f_{i}=f_{i 1}-f_{i 0}=0.71 \times 2 \times 10^{-48} \frac{\left(10^{-1}\right)^{2}-\left(10^{-1}+10^{-15}\right)^{2}}{\left(10^{-1}\right)^{2}\left(10^{-1}+10^{-15}\right)^{2}} \\
=0.71 \times 2 \times 10^{-48} \frac{\left(10^{-1}\right)^{2}-\left(10^{-1}\right)^{2}-2 \times 10^{-1} \times 10^{-15}-10^{-30}}{10^{-2}\left(10^{-2}+2 \times 10^{-1} \times 10^{-15}+10^{-30}\right)} \\
\Delta f_{i}=f_{i 1}-f_{i 0} \approx 0.71 \times 2 \times 10^{-48} \times \frac{-2 \times 10^{-1} \times 10^{-15}}{10^{-2} \times 10^{-2}} \\
=-0.71 \times 2 \times 2 \times 10^{-48} \times \frac{10^{-16}}{10^{-4}}=-2.84 \times 10^{-60} \mathrm{~N}
\end{gathered}
$$


The total force of interaction $F_{P}$ upon an MP, given by the net percussions $\Delta f_{i}$ of all the ECs around it, can be calculated from Equations (4a) and (9c).

$$
F_{P}=n \times \Delta f_{i}=-10^{24} \times 2.84 \times 10^{-60}=-2.84 \times 10^{-36} \mathrm{~N}
$$

For comparison, we will calculate the classical Coulomb force $F_{C}$ using Equation (1) of [1]. In a situation in which MP2 has a charge $+e$ and MP1 has a charge $-e$, located at a laboratory distance of $l=10^{-1} \mathrm{~m}$, this results in

$$
F_{c}=-\frac{(1 e)^{2}}{4 \pi \times 8.85 \times 10^{-12} \times l^{2}}=-\frac{\left(1.6 \times 10^{-19}\right)^{2}}{4 \pi \times 8.85 \times 10^{-12} \times\left(10^{-1}\right)^{2}}=-2.30 \times 10^{-26} \mathrm{~N}(10 \mathrm{a})
$$

It is noted that, in Equations (10) and (10a), the two $F_{P}$ and $F_{C}$ forces obtained were not equal, but were in the same large domain, with $F_{P} \ll F_{C}$, which is also due to the approximations of the physical parameters of ether and the simplified forces $f_{i}$ used in the above numerical calculation and which is more suitable for astronomical distances.

Comparing this result with the result from i), we conclude that the method utilized above and also in [3], is more suitable for the astronomical domain, while for the laboratory and atomic domains, it must be improved.

This can also be seen by considering that the value of $F_{P}$ obtained in (10) is the force resulting only from only one electric interaction path $l_{i}$ from among many interaction paths and multiple corresponding forces $F_{P p}$, as shown in Section 5.

\section{Presentation of the Mechanism of the Physical Interaction between Microparticles with Electric Charges}

We will now analyse the physical mechanism for producing an electrical interaction between the MPs, which we consider as being the only carriers of the positive and negative electric charges. This hypothesis implies the existence of only charged $\mathrm{MP}^{+}$and $\mathrm{MP}^{-}$microparticles, but in this paper the signs + and - will not be indicated but only supposed. Even particles which are considered neutral, such as neutrons, are composed of $\mathrm{MP}^{+}$and $\mathrm{MP}^{-}$pairs, giving zero total charge.

As a result of the electrical interactions between MPs in the ether, there will be a force that will act on each electrically charged MP. Classically, this force is considered today to be Coulomb's $F_{C}$ electric force.

However, this force was recently identified and named by the present authors [3] the Coulomb completed force or $F_{C C}$

The $F_{C C}$ will be created as an effect of $\varepsilon$ and $\gamma$ ether deformations developed on the surface of the MP2, produced by the FVs originating from MP1. We can assume that the FVs are transmitted through ether, by rays, roads or paths, starting from MP1 and inclined at different angles $\alpha_{i}$ (Figure 4). Next, we will denote by $l_{i}$ the lengths of the complete paths between MP1 and MP2, starting at radius $r_{i}$

The effect of $\varepsilon$ and $\gamma$ ether deformations is also seen by changes in the stresses $\sigma$ and $\tau$ acting on the surface of MP2 (Figure 2, Figure 3), which create $F_{C C}$ ac- 
cording to the mechanism presented in Section 2.

The FVs travel at a speed $c_{f}$ with the first FVs reaching MP2 being those that travel the shortest direct path $l_{0}$, arriving after time $t_{0}$ (Figure 4, Figure 5).

$$
t_{0}=l_{0} / c_{f}
$$

The first FVs will leave MP1 at time $t_{i}$. For simplicity, we adopt $t_{i}=0$, without thereby affecting the generality of the analysis. These FVs create $\varepsilon$ and $\gamma$ (both with pulsation $\omega$ ) deformations of the ether cell network, on the surface of MP2 (Figure 2, Figure 4, Figure 5).

Similarly, the FVs travelling along path $l_{1}>I_{0}$ reach MP2 after time $t_{1}$.

$$
t_{1}=l_{1} / c_{f}
$$

Meanwhile, the FVs that travel along path $I_{2}>I_{1}$ arrive after time $t_{2}$

$$
t_{2}=l_{2} / c_{f}
$$

while the FVs that travel along path $I_{n}>I_{n-1}$ arrive after the time $t_{n}$.

$$
t_{n}=l_{n} / c_{f}
$$

However, at the same time $t_{0}$, all the FVs travelling in the directions of rays $r_{1}, r_{2}, r_{3}, \cdots, r_{n}$ and moving along paths $l_{1}, l_{2}, l_{3}, \cdots, l_{n}$, will be able to reach MP2 (Figure 5, Figure 6). However, they left MP1 in the moments preceding $t_{0}$ and their paths must observe the condition of the total accumulated intervals $\Delta I_{t p}$ given that we also have:

$$
\Delta t=\Delta l / c_{f} \text {, and hence (11d) }
$$

However, each of these $\mathrm{FV}_{i}$ vibrations creates a percussion force $F_{P}$ on MP2, according to Equation (6) in Section 3.

All these $F_{P}$ forces, when summed up over all $\mathrm{FV}_{\mathrm{i}}$ vibrations on the entire surface $S$ of MP2, create the Coulomb completed force $F_{C O}$ according to our hypothesis in Equation (21) from [3].

We can depict the elementary force of the $F_{P}$ percussion type in a simple and natural general form similar to Equation (6), as a variation proportional to the inverse of the square root of the ray $r$ travelled by the FVs in ether free of any other EPs.

$$
F_{P}=\frac{k_{p}}{r^{2}}
$$

At time $t_{0}$, all the $F_{P_{i}}$ forces created by FVs on MP2, leaving MP1 at times $t_{i}$ from Equation (11c), preceding $t_{0}$ and corresponding to the lengths $I_{i}$ of the travelled paths, simultaneously act directly on MP2. The different forces $F_{P i}$ given by (12) oriented according to the rays $r_{i}$ inclined at angles $\alpha_{i}$ are as follows (Figure 5, Figure 6).

$$
F_{P 0}=\frac{k_{p}}{l_{0}^{2}} ; F_{P 1}=\frac{k_{p}}{l_{1}^{2}} ; F_{P 2}=\frac{k_{p}}{l_{2}^{2}} ; \cdots ; F_{P i}=\frac{k_{p}}{l_{i}^{2}} ; \cdots ; F_{P n}=\frac{k_{p}}{l_{n}^{2}}
$$




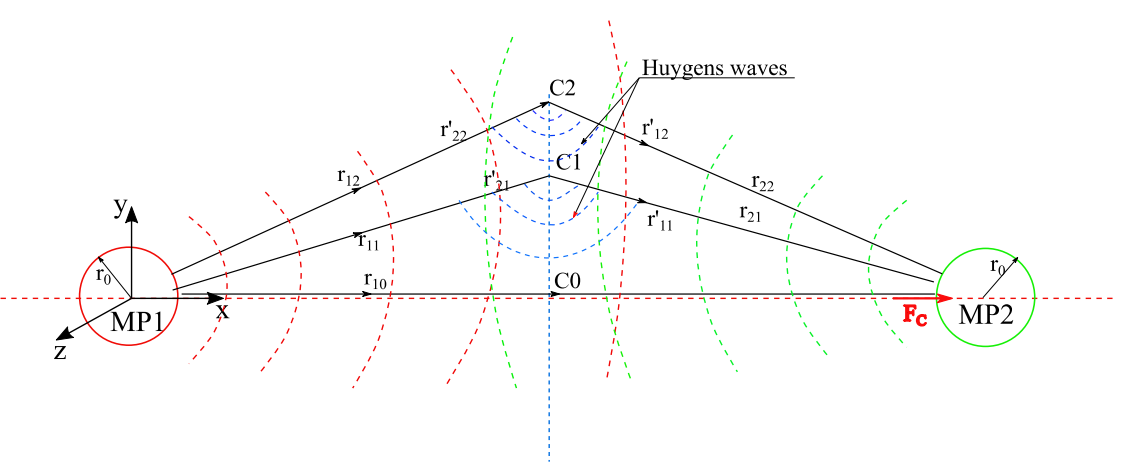

Figure 5. Transmission of FVs in the form of rays $r_{i}$ created by MP1 and MP2, which at points $C$ continue as secondary waves of the Huygens type, in the $O x y z$ reference frame attached to the immovable MP1.

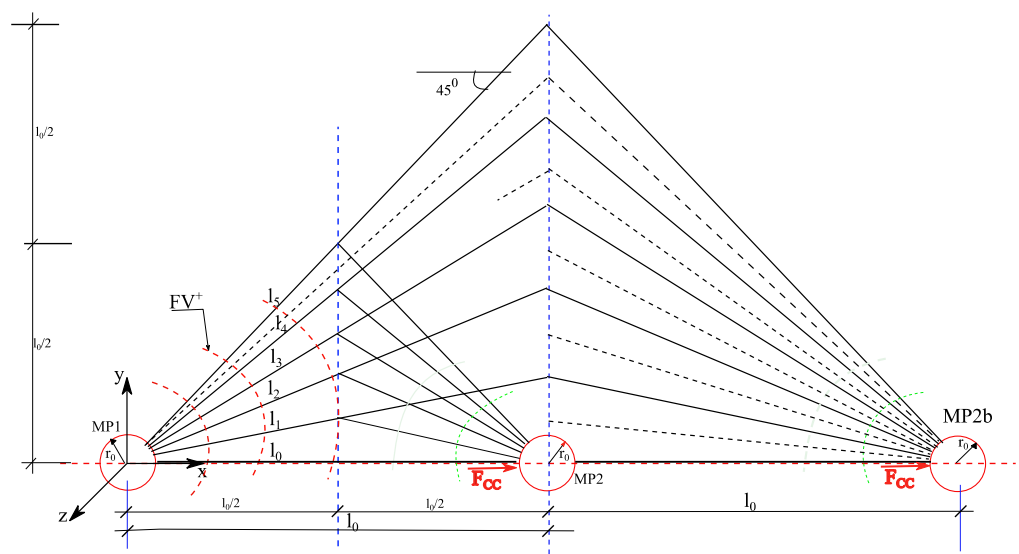

Figure 6. Simple representation of the triangular pathways $l_{i}$ of the $F_{C C}$ electrical interaction force between MP1, MP2 and MP2b, including $2 I_{0}$ with 11 paths, in the Oxyz reference frame attached to the immovable MP1.

The total force $F_{P}^{\text {tot }}$ is obtained by summing all the $F_{P i}$ forces from Equations (13), as they are simultaneous actions with simultaneous percussions $p_{i}$ in time. However, the sum must be vectorial.

This real $F_{P}^{\text {tot }}$ total force will only be obtained correctly if each force $F_{P i}$ in the series of forces in (13) is of the $F_{P i}$ type, i.e., is the result of a single full vibration $\mathrm{FV}_{\mathrm{i}}$, which will produce a single set of percussions $p_{i}$. This result can only be obtained if, when starting from MP1, the $\mathrm{FV}_{\mathrm{i}}$ waves are separated in time by a time period $T_{i}$. This fact will correspond, on arrival at MP2, with the distance between two successive wavelengths of $\mathrm{FV}_{\mathrm{i}}$.

$$
\Delta l_{i}=\lambda=c t
$$

On MP2b, the $F_{2 i}$ forces have the same formulas as in Equation (13), but with new distances $l_{i}$ adapted according to Figure 6 .

Below, we analyse the situation created at the surface of an MP2, where the specific deformations $\varepsilon$ and $\gamma(\omega)$ occur in the EC network of the ether. These deformations are due to the FVs created by a series of microparticles MP1a, MP1b, MP1c, ... (Figure 7). 


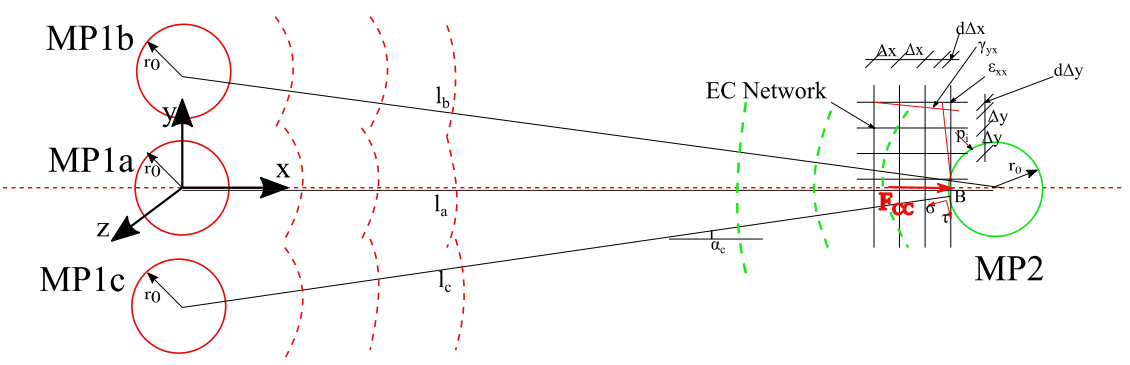

Figure 7. The mode of occurrence of the stresses $\sigma$ and $\tau$ on MP2, due to strains $\varepsilon$ and $\gamma(\omega)$ created by MP1a, in the Oxyz reference frame attached to the immovable MP1.

Obviously, according to Figure 7, the deformations $\varepsilon$ and $\gamma(\omega)$ in the ether, at a point $\mathrm{P}_{\mathrm{i}}$ on the surface of MP2, located at distances $l_{a}, l_{b}, l_{c}$ from MP1a, MP1b and MP1c, decrease inversely relative to the square of these distances, when taking into account the formula for $F_{C}$ obtained in Equation (1) from [1] and the formula of the $F_{P}$ force obtained in Equation (13). This behaviour of FVs in the ether, considered as a special mechanical matter, is also justified by its similarity to a common physical phenomenon depending on $1 / r^{2}$ in normal matter (solid, liquid or gas).

Therefore, the unitary forces $\sigma$ and $\tau$, exerted upon MP2 by the ECs due to the strains $\varepsilon$ and $\gamma(\omega)$ created by MP1a, MP1b, .., will have to be inversely proportional to the square of these distances $l_{a}^{2}, \cdots, l_{c}^{2}, \cdots$ based on the result from Section 4 .

In Figure 7, only the effect of the direct rays $r_{a}$, having the slope $\alpha_{0}=0^{\circ}$ with path length $l_{\mathrm{a}}$, is represented as a grid of ECs near MP2 and as percussions $p_{i}$.

However, it is necessary to analyse the effects upon MP2 produced by other possible indirect paths starting from MP1, on various radii $r_{1 i}$ drawn in any direction with inclination angles $\alpha_{i}$ (Figures 5-7).

As the rays starting from MP1 are inclined at angle $\alpha_{i}$ they are able to reach MP2 on an indirect path in the form of an isosceles triangle (Figure 6). This isosceles path will be the shortest path starting from MP1 at any angle $\alpha_{i}$.

Such a path can be accomplished physically by applying the Huygens principle to the midpoint $C_{i}$ of the radius $r_{1 i}$ (Figure 5). Here, the circles (in the plane problem, but real spheres in the spatial problem) of the Huygens secondary waves with a centre at $C_{i}$ will allow the construction of the symmetrical radius $r_{1 i}^{\prime}$ of the radius $r_{1 i}$.

In fact, at the point $C_{i}$ (Figure 5), the waves which will travel along the ray $r_{1 p}$, will arrive at the same time as those arriving from the $r_{2 i}$ radius that started from MP2 simultaneously with $r_{1:}$ As a result, the interaction between MP1 and MP2 must be symmetrical in space and in time (Figure 5, Figure 6).

Therefore, it can also be considered that, at point $\mathrm{C}_{\mathrm{i}}$ (Figure 5), a special interference of FVs appears, with no terminus point between the waves in the rays $r_{1 i}$ and $r_{2 i}$. At this point, these rays mutually interchange their path, moving in the forward direction beyond point $C_{i}$ This phenomenon is symmetrical due to the symmetry of MP1 and MP2, including the ether homogeneity and hence the 
symmetry of HM16.

The rays $r_{i}+r_{i}^{\prime}$, which are inclined at the angle $\alpha_{i}$, will travel the corresponding distances $l_{i}$ (Figure 5, Figure 6) to MP2 in a longer time $t_{i}$.

However, at a certain moment $\left(t_{0}\right)$, various waves with rays $r_{i}$, which left MP1 at various moments preceding $t_{0}$ (the shortest time), will arrive at MP2 with forces $F_{P i}$ according to Equation (13), giving a total force $F_{P}^{\text {tot }}$.

A question arises as to whether the overlapping of rays with various angles $\alpha_{i}>$ 0 from the other MPs existing in the range close to MP2 (Figure 6), influence the final result of the interaction force $F_{P}$ between MP1 and MP2. The situation occurs in compact MBs (solids, liquids) when the distance $d_{0}$ between neighbouring MPs is less than $1_{0}$ at a certain density.

However, as we discuss and analyse for two different physical situations for MP2, isolated or not isolated, the result for the $F_{P}$ force must be different. Consequently, it is necessary to introduce a correction reducing $F_{P}$ based on the MP density. Such a correction is possible using various mathematical procedures, including correcting the power of 2 in the distance $P$.

However, in our current stage of analysis, we evaluate the interaction between any MP1 and MP2 as isolated, considering the normal contribution of all the rays with angles $\alpha \leq 45^{\circ}$ (a cone, in space).

\section{Presentation of the Calculation for the $F_{P}$ Electric Interaction Force between Microparticles, Considering Multiple Paths in the Ether, including Justification of the Term $\ln r$ in $F_{C C}$ Force}

\subsection{The General Case of the Electric Interaction Problem}

A calculus demonstrating the capping trend of decreasing of the $F_{P}$ force with distance $l$ (according to the term $\ln r$ ), is presented in the following analysis. Here, we analyse the variation of electric force $F_{P}$ with distance, starting from the percussions of the ether (Sections 4 and 5). The next calculation of $F_{P}$ is based on multiple paths $l_{i}$ of FVs between two MPs.

We consider the situation of two MPs, MP1 and MP2, each with an elementary electric charge $\pm q$, located at a direct distance $l_{0}$ (Figure 8 ).

The electrical interaction between the two MPs occurs via all FVs, which can occur outside the direct path $l_{0}$ and travel on other deviating paths, with the general form of concave curves (Figure 8).

As a simplified particular case, we adopt here simple paths in the form of isosceles triangles, as discussed in Section 5.

In the general case, we accept that we have discretized the area of influence between MP1 and MP2 in a number $n+1$ of curvilinear paths $l_{i}$ (Figure 8 ).

These paths are arranged on one half of the interaction surface, which is bounded at the bottom by the Ox axis and at the top by the line inclined at $45^{\circ}$, in the case of the plane problem. Figure 8 presents the case with 6 paths (red), $l_{0}$, $I_{1}, l_{2}, I_{3} I_{4}$, and $I_{5}$, which applies to the plane problem of the interaction between MP1 and MP2. 


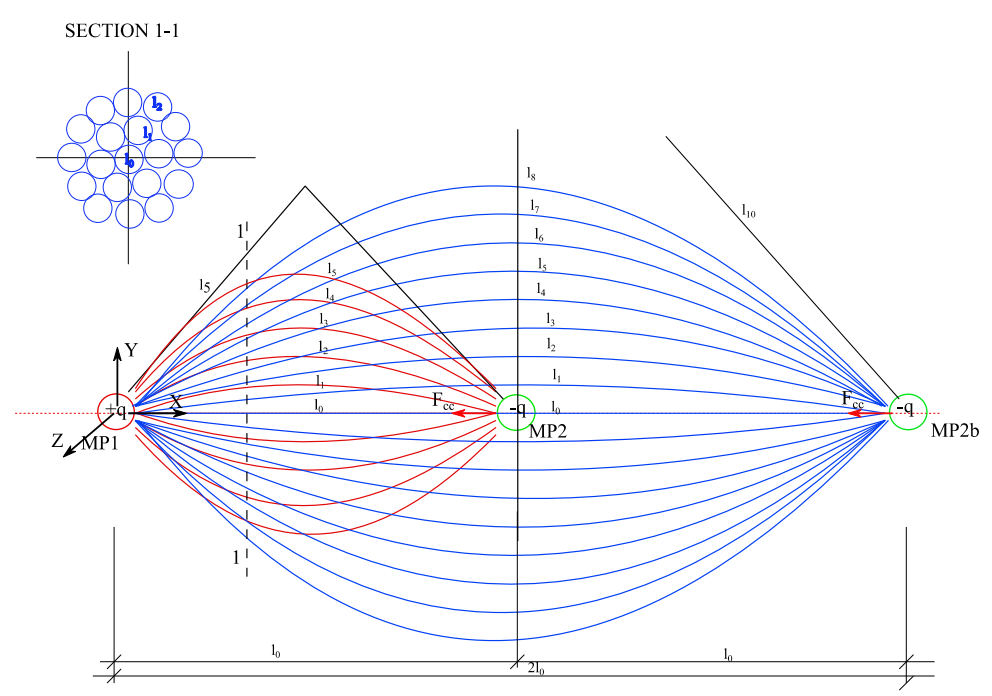

Figure 8. Representation in the HM16A ether of the concave-shaped paths, approximated by isosceles triangles, for the electrical interaction force $F_{P}$, which creates the $F_{C C}$ force between MP1 and MP2, in the Oxyz reference frame attached to the immovable MP1.

Further, in the general case with $n+1$ paths $l_{0}, l_{1}, l_{2}, l_{3}, \cdots, l_{n}$, for the total force from the percussions $F_{P}^{\text {tot }}$, noting the differences in the paths $\Delta l_{i}=l_{i}-l_{i-1}$, we have from Equation (13):

$$
F_{P}^{\text {tot }}=\frac{k_{p}}{l_{0}^{2}}\left[1+\frac{1}{\left(1+\frac{\Delta l_{1}}{l_{0}}\right)^{2}}+\frac{1}{\left(1+\frac{\Delta l_{1}+\Delta l_{2}}{l_{0}}\right)^{2}}+\cdots+\frac{1}{\left(1+\frac{\Delta l_{1}+\Delta l_{2}+\cdots+\Delta l_{n}}{l_{0}}\right)^{2}}\right]
$$

For simplicity, we consider the length differences of paths $\Delta l$ to be constant and identical between any two paths $l_{i}$ and $l_{i-1}$. This does not significantly affect the final result. These paths differences $\Delta l$ must be at least equal to the wavelength $\lambda$, according to Section 5, but may be multiples of it, as in the general case.

In turn, the lengths of successive paths $l_{i}$ can be written as:

$$
l_{i}=l_{0}+i \Delta l \text { with } i \in(0, n)
$$

In this case, we can also write:

$$
\Delta l=l_{i}-l_{i-1}=\frac{l_{n}-l_{0}}{n}
$$

We will define the relative length difference $\varepsilon$ as follows:

$$
\varepsilon=\frac{\Delta l}{l_{0}}
$$

We will directly calculate the magnitude of the total corrected force $F_{P}^{\text {tot }}$, adding, term by term, the $F_{P i}$ forces, which arise from the various $\mathrm{FV}$ waves that have passed along the paths $I_{i}$ (Figure 6, Figure 8), according to Equations (7) 
and (13), resulting in:

$$
\begin{aligned}
F_{P}^{\text {tot }}=\frac{k_{p}}{l_{0}^{2}}\left[1+\frac{1}{\left(1+\frac{\Delta l}{l_{0}}\right)^{2}}+\frac{1}{\left(1+\frac{2 \Delta l}{l_{0}}\right)^{2}}+\cdots+\frac{1}{\left(1+\frac{i \Delta l}{l_{0}}\right)^{2}}+\cdots+\frac{1}{\left(1+\frac{(n) \Delta l}{l_{0}}\right)^{2}}\right] \\
F_{P}^{\text {tot }}=\frac{k_{p}}{l_{0}^{2}}\left[1+\frac{1}{(1+\varepsilon)^{2}}+\frac{1}{(1+2 \varepsilon)^{2}}+\cdots+\frac{1}{(1+i \varepsilon)^{2}}+\cdots+\frac{1}{(1+(n) \varepsilon)^{2}}\right]
\end{aligned}
$$

It is noted that, in Equation (17), after the first term, there appears a series with $n$ terms with the variable ratio $q$, where

$$
q=\left(\frac{1+(k-1) \varepsilon}{1+k \varepsilon}\right)^{2} \text { and the first term is } a_{1}=\frac{1}{(1+\varepsilon)^{2}} ; k \in(2,3, \cdots, n)
$$

The sum of this series is too laborious to compute analytically.

Consequently, we will apply the numerical calculation method to the series sum, whose results can then be generalized as follows.

\subsection{Case of the Plane Problem for Numerical Calculations}

\subsubsection{Situation with the Maximum Angle $\alpha=45^{\circ}$}

\section{a.1) Situation Involving the Initial Distance $I_{0}$, Considering the Upper} Half of the Plan Domain, Comprising 6 Paths

In this section, we use an approximate numerical empirical calculation by considering some numerical cases with a small number of paths $(n+1)$. The result obtained via this procedure can be then generalized and will be useful at the current stage of the HM16 model.

In the first situation a.1) (in the parentheses in (17)), we consider the initial case (Figure 8) in which $n=5$ and there are 6 paths which are distributed initially on only half of the domain above the $O x$ axis, in the plane problem. For simplicity, we replace the curved paths in Figure 8 with isosceles triangular paths, according to Section 5, as shown in Figure 6 and Figure 9. In this case, for the angle $\alpha_{\max }=45^{\circ}$ from Equation (16a), we obtain:

$$
\begin{gathered}
\delta l=l_{5}-l_{0}=1.41 l_{0}-l_{0}=0.41 l_{0} \\
\Delta l=\frac{\delta l}{n} \\
\varepsilon=\frac{\Delta l}{l_{0}}=\frac{0.41 l_{0}}{l_{0} \cdot n}=\frac{0.41}{5}=0.082
\end{gathered}
$$

By introducing (21) into Equation (17), we have:

$$
\begin{aligned}
F_{P}^{\text {tot }}= & \frac{k_{p}}{l_{0}^{2}}\left[1+\frac{1}{(1+0.082)^{2}}+\frac{1}{(1+2 \times 0.082)^{2}}+\frac{1}{(1+3 \times 0.082)^{2}}\right. \\
& \left.+\frac{1}{(1+4 \times 0.082)^{2}}+\frac{1}{(1+5 \times 0.082)^{2}}\right]
\end{aligned}
$$




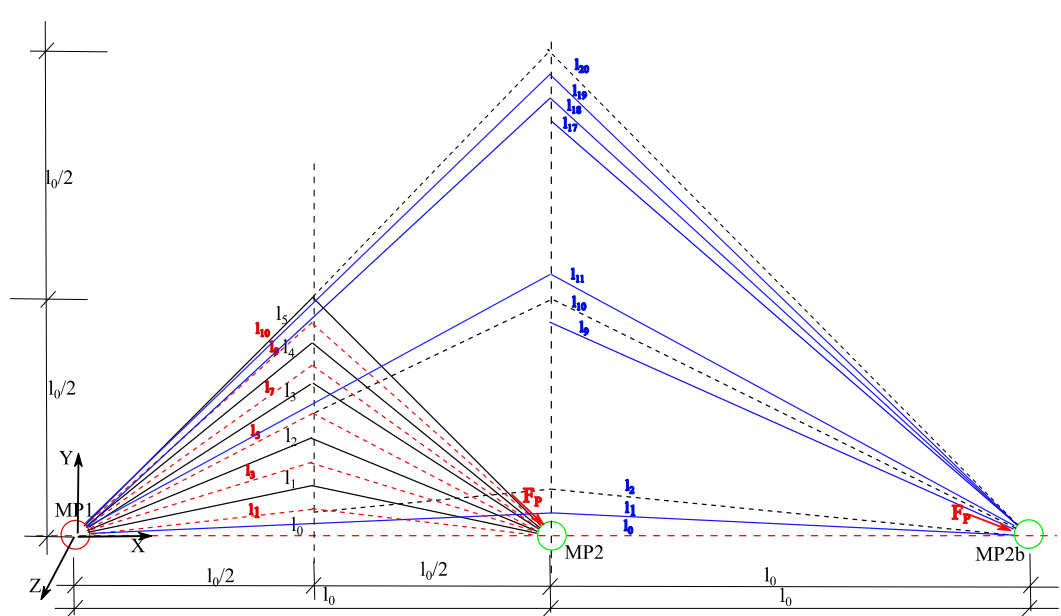

Figure 9. Representation of the triangular paths $l_{i}$ of the electrical interaction force $F_{P}$, which creates the $F_{C C}$ force between MP1 and MP2, including the case $2 I_{0}$ with 21 paths, in the $O x y z$ reference frame attached to the immovable MP1.

$$
\begin{aligned}
F_{P}^{\text {tot }} & =\frac{k_{p}}{l_{0}^{2}}[1+0.854+0.783+0.644+0.567+0.503] \\
& =\frac{k_{e}}{l_{0}^{2}}[1+3.35]=\frac{k_{e}}{l_{0}^{2}} \times 4.35
\end{aligned}
$$

a.1).s. A supplemental calculus (not reproduced here) also for this distance but with 9 paths gave the result:

$$
F_{P}^{\text {tot }}=\frac{k_{e}}{l_{0}^{2}}(1+5.432)=\frac{k_{e}}{l_{0}^{2}} \times 6.432
$$

a.2) Situation Involving the Initial Distance $I_{0}$, on Half of the Plan Domain, with 11 Paths

Now, we double the number of paths by thickening the 6 paths considered in a.1), according to Figure 9, including the dashed paths, resulting in 11 paths for which $n=10$. For simplicity, we have also replaced the curved paths with isosceles triangles, according to Section 5. In this case, for the angle $\alpha_{\max }=45^{\circ}$ from (16b), we obtain:

$$
\begin{gathered}
\delta l=l_{10}-l_{0}=1.41 l_{0}-l_{0}=0.41 l_{0} \\
\varepsilon=\frac{\Delta l}{l_{0}}=\frac{0.41 l_{0}}{l_{0} \cdot 10}=\frac{0.41}{10}=0.041
\end{gathered}
$$

By introducing (23a) into Equation (17), we obtain:

$$
\begin{aligned}
F_{P}^{\text {tot }}= & \frac{k_{p}}{l_{0}^{2}}\left[1+\frac{1}{(1+0.041)^{2}}+\frac{1}{(1+2 \times 0.041)^{2}}+\frac{1}{(1+3 \times 0.041)^{2}}\right. \\
& +\frac{1}{(1+4 \times 0.041)^{2}}+\frac{1}{(1+5 \times 0.041)^{2}}+\frac{1}{(1+6 \times 0.041)^{2}} \\
& \left.+\frac{1}{(1+7 \times 0.041)^{2}}+\frac{1}{(1+8 \times 0.041)^{2}}+\frac{1}{(1+9 \times 0.041)^{2}}+\frac{1}{(1+10 \times 0.041)^{2}}\right]
\end{aligned}
$$




$$
\begin{aligned}
F_{P}^{\text {tot }}= & \frac{k_{p}}{l_{0}^{2}}[1+0.9227+0.8541+0.793+0.738+0.6886 \\
& +0.644+0.6037+0.567+0.5335+0.503] \\
= & \frac{k_{p}}{l_{0}^{2}}[1+6.847] \\
= & \frac{k_{p}}{l_{0}^{2}} \times 7.847
\end{aligned}
$$

\section{b.1) Situation with the Initial Distance Doubled $\left(2 I_{0}\right)$ on Half of the Plan} Domain, with 6 Paths

In the second case in Figure 9, between MP1 and MP2 we have a distance $2 I_{0}$ and $n=5$ with 6 paths, so we replace $I_{0}$ with $2 I_{0}$ in Equations (16), (17) and (21), resulting in:

$$
\begin{gathered}
l_{5}-l_{0}=1.41 \times 2 l_{0}-2 l_{0}=0.41 \times 2 l_{0}=0.82 l_{0} \\
\Delta l=\frac{0.82 l_{0}}{5}=0.164 l_{0} \\
\varepsilon=\frac{\Delta l}{2 l_{0}}=\frac{0.164 l_{0}}{2 l_{0}}=0.082
\end{gathered}
$$

By introducing (26b) into Equation (17), we obtain:

$$
\begin{gathered}
F_{P}^{\text {tot }}=\frac{k_{p}}{\left(2 l_{0}\right)^{2}}\left[1+\frac{1}{(1.082)^{2}}+\frac{1}{(1.164)^{2}}+\frac{1}{(1.246)^{2}}+\frac{1}{(1.328)^{2}}+\frac{1}{(1.41)^{2}}\right] \\
F_{P}^{\text {tot }}=\frac{k_{p}}{\left(2 l_{0}\right)^{2}}[1+0.854+0.738+0.644+0.567+0.503] \\
F_{P}^{\text {tot }}=\frac{k_{p}}{\left(2 l_{0}\right)^{2}}[1+3.306]=\frac{k_{p}}{\left(2 l_{0}\right)^{2}} \times 4.306 \\
=\frac{k_{p}}{\left(2 l_{0}\right)^{2}} \frac{1}{4} \times 4.306=\frac{k_{p}}{l_{0}^{2}} \times 1.0765
\end{gathered}
$$

b.2) Situation with the Initial Distance Doubled $\left(2 I_{0}\right)$ on Half of the Plan

\section{Domain, with 11 Paths}

Now, we double the number of paths by thickening the 6 paths considered in b.1) according to Figure 9, including the dashed paths, resulting in 11 paths for which $n=10$. For simplicity, we also replace the curved paths from Figure 7 with isosceles triangles from Figure 9, according to Section 5. In this case, for the angle $\alpha_{\max }=45^{\circ}$, we obtain from (26a):

$$
\begin{gathered}
l_{10}-l_{0}=1.41 \times 2 l_{0}-2 l_{0}=0.41 \times 2 l_{0}=0.82 l_{0} \\
\Delta l=\frac{0.82 l_{0}}{10}=0.082 l_{0} \\
\varepsilon=\frac{\Delta l}{2 l_{0}}=\frac{0.082 l_{0}}{2 l_{0}}=0.041
\end{gathered}
$$

Introducing (28b) into Equation (17), we have: 


$$
\begin{gathered}
F_{P}^{\text {tot }}=\frac{k_{p}}{\left(2 l_{0}\right)^{2}}\left[1+\frac{1}{(1+0.041)^{2}}+\frac{1}{(1+2 \times 0.041)^{2}}+\frac{1}{(1+3 \times 0.041)^{2}}\right. \\
+\frac{1}{(1+4 \times 0.041)^{2}}+\frac{1}{(1+5 \times 0.041)^{2}}+\frac{1}{(1+6 \times 0.041)^{2}} \\
+\frac{1}{(1+7 \times 0.041)^{2}}+\frac{1}{(1+8 \times 0.041)^{2}}+\frac{1}{(1+9 \times 0.041)^{2}} \\
\left.\quad+\frac{1}{(1+10 \times 0.041)^{2}}\right] \\
F_{P}^{\text {tot }}=\frac{k_{p}}{\left(2 l_{0}\right)^{2}}[1+0.9227+0.8541+0.793+0.738+0.6886 \\
+0.644+0.6037+0.567+0.5335+0.503] \\
=\frac{k_{p}}{\left(2 l_{0}\right)^{2}}[1+6.847]=\frac{k_{p}}{\left(2 l_{0}\right)^{2}} \times 7.847=\frac{k_{p}}{\left(l_{0}\right)^{2}} \frac{1}{4} \times 7.847=\frac{k_{p}}{\left(l_{0}\right)^{2}} \times 1.962
\end{gathered}
$$

b.2).s. A supplemental calculus (not reproduced here) also for this distance but with 17 paths gave the result:

$$
F_{P}^{t o t}=\frac{k_{e}}{l_{0}^{2}}(1+2.045)=\frac{k_{e}}{l_{0}^{2}} \times 3.045
$$

b.3) Situation with the Initial Distance Doubled (2 $\left.I_{0}\right)$ on Half of the Plan Domain, with 21 Paths

Now, we double the number of paths by thickening the 6 paths considered in b.2) according to Figure 9, including the dashed paths, resulting in 21 paths for which $n=20$. For the sake of simplicity, we also replace the curved paths with isosceles triangles, according to Section 5. In this case, for the angle $\alpha_{\max }=45^{\circ}$, from (28a) we obtain:

$$
\begin{gathered}
l_{20}-l_{0}=1.41 \times 2 l_{0}-2 l_{0}=0.41 \times 2 l_{0}=0.82 l_{0} \\
\Delta l=\frac{0.82 l_{0}}{20}=0.041 l_{0} \\
\varepsilon=\frac{\Delta l}{2 l_{0}}=\frac{0.041 l_{0}}{2 l_{0}}=0.0205
\end{gathered}
$$

By introducing (30b) into Equation (17), we have:

$$
\begin{aligned}
F_{P}^{\text {tot }}= & \frac{k_{p}}{\left(2 l_{0}\right)^{2}}\left[1+\frac{1}{(1+0.0205)^{2}}+\frac{1}{(1+2 \times 0.0205)^{2}}+\frac{1}{(1+3 \times 0.0205)^{2}}\right. \\
& +\frac{1}{(1+4 \times 0.0205)^{2}}+\frac{1}{(1+5 \times 0.0205)^{2}}+\cdots+\frac{1}{(1+16 \times 0.0205)^{2}} \\
& +\frac{1}{(1+17 \times 0.0205)^{2}}+\frac{1}{(1+18 \times 0.0205)^{2}}+\frac{1}{(1+19 \times 0.0205)^{2}} \\
& \left.+\frac{1}{(1+20 \times 0.0205)^{2}}\right]
\end{aligned}
$$




$$
\begin{aligned}
F_{P}^{\text {tot }}= & \frac{k_{p}}{\left(2 l_{0}\right)^{2}}[1+0.960+0.9227+0.887+0.8541+0.8227+0.793 \\
& +0.7647+0.738+0.7126+0.6886+0.6658+0.644+0.6234 \\
& +0.6037+0.5849+0.567+0.5499+0.5335+0.5179+0.503](31 \mathrm{a}) \\
= & \frac{k_{p}}{\left(2 l_{0}\right)^{2}}[1+13.271]=\frac{k_{p}}{\left(2 l_{0}\right)^{2}} \times 14.271 \\
= & \frac{k_{p}}{\left(l_{0}\right)^{2}} \frac{1}{4} \times 14.271=\frac{k_{p}}{\left(l_{0}\right)^{2}} \times 3.318
\end{aligned}
$$

c.1) Situation with the Initial Distance $I_{0}$ and the Path Number Symmetrically Doubled across the Whole Plan Area

Let us now consider the complete plane situation shown in Figure 9, including the symmetrical paths relative to the horizontal centre axis $O x$. This is a plane problem, doubling the terms in cases a) and b) above, which results in the following cases.

Case a.1):

$$
F_{P}^{\text {tot }}=\frac{k_{p}}{l_{0}^{2}}[1+3.35+3.35]=\frac{k_{p}}{l_{0}^{2}}[1+6.70]=\frac{k_{p}}{l_{0}^{2}} \times 7.70
$$

Case a.2):

$$
F_{P}^{\text {tot }}=\frac{k_{p}}{l_{0}^{2}}[1+6.847+6.847]=\frac{k_{p}}{l_{0}^{2}}[1+13.694]=\frac{k_{p}}{l_{0}^{2}} \times 14.694
$$

Case b.1):

$$
F_{P}^{\text {tot }}=\frac{k_{p}}{4 l_{0}^{2}}[1+3.306+3.306]=\frac{k_{p}}{4 l_{0}^{2}}[1+6.612]=\frac{k_{p}}{l_{0}^{2}} \times 1.9034
$$

Case b.2):

$$
F_{P}^{\text {tot }}=\frac{k_{p}}{4 l_{0}^{2}}[1+6.847+6.847]=\frac{k_{p}}{4 l_{0}^{2}}[1+13.694]=\frac{k_{p}}{l_{0}^{2}} \times 3.673
$$

Case b.3):

$$
F_{P}^{\text {tot }}=\frac{k_{p}}{4 l_{0}^{2}}[1+13.271+13.271]=\frac{k_{p}}{4 l_{0}^{2}}[1+26.542]=\frac{k_{p}}{l_{0}^{2}} \times 6.885
$$

Now, in the relations (32), (33), (34), (35) and (36), it can be observed that the coefficients multiplying the same fraction $k_{p} / l_{0}^{2}$ are almost doubled, though not exactly but with a slightly diminished value, compared to similar cases in the relations (22a), (25), (27a), (29a) and (31a), as set out in Section 6.2.1 above. Accordingly, the favourable effect of the duplication of paths is acknowledged in this subsection.

\subsubsection{Situation with the Angle $\alpha=60^{\circ}$}

d.1) Case of the Plane Domain Half Restricted: Path $I_{0}$, from Case a.1)

We now consider the case in which $\alpha=60^{\circ}$. We add four additional paths giving $6+4=10$ paths from 6.2.1. The number of intervals will be $n=10-1=$ 
9.

$$
\begin{gathered}
\delta l=l_{10}-l_{0}=2 l_{0}-l_{0}=l_{0} \\
\Delta l=\delta l / n=l_{0} / 9=0.111 l_{0} ; \quad \varepsilon=\Delta l / l_{0}=0.111
\end{gathered}
$$

We introduce (37a) into Equation (17), resulting in:

$$
\begin{aligned}
F_{P}^{\text {tot }}= & \frac{k_{p}}{l_{0}^{2}}\left[1+\frac{1}{(1+0.111)^{2}}+\frac{1}{(1+2 \times 0.111)^{2}}+\frac{1}{(1+3 \times 0.111)^{2}}\right. \\
+ & \left.\frac{1}{(1+4 \times 0.111)^{2}}+\frac{1}{(1+5 \times 0.111)^{2}}+\cdots+\frac{1}{(1+9 \times 0.111)^{2}}\right] \\
F_{P}^{\text {tot }}= & \frac{k_{p}}{l_{0}^{2}}[1+0.810+0.6696+0.5628+0.4795+0.4135 \\
& +0.3603+0.3166+0.2805+0.246] \\
= & \frac{k_{e}}{l_{0}^{2}}[1+4.1388]=\frac{k_{e}}{l_{0}^{2}} \times 5.1388
\end{aligned}
$$

The increase in the coefficient will be $5.1388 / 4.35=1.18=118 \%$. Accordingly, the favourable effect of increasing the angle $\alpha$ to $60^{\circ}$ is confirmed.

d.2) Case of the Half Restricted Domain: Path $21_{0}$, from Case b.2)

We now consider the case in which $\alpha=60^{\circ}$. We add nine additional paths giving $10+9=19$ paths from 6.2.1. The interval number is $n=19-1=18$.

$$
\begin{gathered}
\Delta l=l_{19}-l_{0}=4 l_{0}-2 l_{0}=2 l_{0} \\
\Delta l=\delta l / n=2 l_{0} / 18=0.111 l_{0} ; \quad \varepsilon=\Delta l / 2 l_{0}=0.111 l_{0} / 2 l_{0}=0.0555
\end{gathered}
$$

We introduce (39a) into Equation (17) resulting in:

$$
\begin{aligned}
F_{P}^{\text {tot }}= & \frac{k_{p}}{\left(2 l_{0}\right)^{2}}\left[1+\frac{1}{(1+0.0555)^{2}}+\frac{1}{(1+2 \times 0.0555)^{2}}+\frac{1}{(1+3 \times 0.0555)^{2}}\right. \\
& \left.+\frac{1}{(1+4 \times 0.0555)^{2}}+\frac{1}{(1+5 \times 0.0555)^{2}}+\cdots+\frac{1}{(1+19 \times 0.111)^{2}}\right] \\
F_{P}^{\text {tot }}= & \frac{k_{p}}{\left(2 l_{0}\right)^{2}}[1+0.8976+0.810+0.7349+0.6696+0.6127+0.5628 \\
& +0.5186+0.4795+0.4447+0.4135+0.3855+0.3603+0.3374 \\
& +0.3166+0.2978+0.2805+0.2647+0.246+0.2369] \\
= & \frac{k_{p}}{\left(2 l_{0}\right)^{2}}[1+8.8696]=\frac{k_{p}}{\left(2 l_{0}\right)^{2}} \times 9.8696 \\
= & \frac{k_{p}}{l_{0}^{2}} \frac{1}{4} \times 9.8696=\frac{k_{p}}{l_{0}^{2}} \times 2.4674
\end{aligned}
$$

The increase in the coefficient will be $2.4674 / 1.962=1.25=125 \%$. Consequently, the favourable effect of increasing the angle $\alpha$ from $45^{\circ}$ to $60^{\circ}$ is confirmed above $(+25 \%)$. But the paths extension beyond $45^{\circ}$ is not justified physically because it do not match with ether symmetry and homogeneity, and will be abandoned. 


\subsection{Case of the Spatial Problem}

\section{a) General Situation}

In order to be able to generalize to the spatial problem from any of the plane problems in Section 6.2, the number of paths which it is possible to consider in the spatial case will be determined.

For this, we note that the central path $I_{0}$ considers the ether as a cylinder of maximum radius $\mathrm{r}_{0}$ in the middle part of the path (Figure 8, left). Further, the second row of cylindrical paths, each having a maximum radius $r_{0}$, is arranged in a circular manner with the cylinders centred on the paths in the form of a circle with radius $r_{1}=2 r_{0}$. The length of this circle is

$$
L_{1}=2 \pi r_{1}=2 \pi\left(2 r_{0}\right)=4 \pi r_{0}
$$

The circles in row 2, which are evenly distributed, will have centres located on the $L_{1}$ circle to which a length reduction of approximately $5 \%$ is applied, to take account of the curvature of the route. Under these conditions, the reduced cylinder diameter will be $d_{r}=0.95 \times 2 r_{0}=1.9 r_{0}$. Meanwhile, the number of adjacent circles (and cylinders) $N_{1}$ will be (Figure 8, left):

$$
N_{1}=\frac{L_{1}}{d_{r}}=\frac{4 \pi r_{0}}{1.9 r_{0}}=\frac{4 \pi}{1.9}=6.61 \mathrm{pcs}
$$

Now, the paths in the third row with radius $\mathrm{r}_{0}$ are placed with the centres of the cylinders on the circle with radius $r_{2}=4 r_{0}$. The length of this circle is (Figure 8 , left):

$$
L_{2}=2 \pi r_{2}=2 \pi\left(4 r_{0}\right)=8 \pi r_{0}
$$

The number of uniformly distributed circles will be:

$$
N_{2}=\frac{L_{2}}{d_{r}}=\frac{8 \pi r_{0}}{1.9 r_{0}}=\frac{8 \pi}{1.9}=13.2 \mathrm{pCs}
$$

Similarly, the paths in the fourth row with radius $r_{0}$ can be distributed with the centres of the cylinders on the circle having a radius $r_{3}=6 r_{0}$. The length of this circle is:

$$
L_{3}=2 \pi r_{3}=2 \pi\left(6 r_{0}\right)=12 \pi r_{0}
$$

The number of adjacent circles, evenly distributed, will be:

$$
N_{3}=\frac{L_{3}}{d_{r}}=\frac{12 \pi r_{0}}{1.9 r_{0}}=\frac{12 \pi}{1.9}=19.8 \text { pcs }
$$

From Equations (42), (44) and (46), we can observe the rule for forming the rows of cylinders, the number of which is an arithmetic progression, with a ratio of approximately 6 pcs (covered for 7 pcs) and having the first term equal to 1 .

b) Particular Situations

e.1) Situation Involving the Initial Distance $I_{0}$ in Space, with 6 Initial Paths (from Case a.1)

We now recalculate the magnitude of the force of interaction $F_{P}$ between MP1 and MP2, considering all spatial paths for the initial distance $I_{0}$, starting from the 
six paths in Section 6.2. The number of these paths (pieces) will be in accordance with the rule above:

$$
N^{\text {tot }}=1+6+13+19+25+31=95 \text { pcs }
$$

The magnitude of the $F_{p}$ interaction force is obtained from (22a) using (47):

$$
\begin{aligned}
F_{P}^{\text {tot }} & =\frac{k_{p}}{l_{0}^{2}}[1 \times 1+0.854 \times 6+0.783 \times 13+0.644 \times 19+0.567 \times 25+0.503 \times 31] \\
& =\frac{k_{p}}{l_{0}^{2}}[1+5.12+10.18+12.23+14.17+15.59]=\frac{k_{e}}{l_{0}^{2}} \times 58.29
\end{aligned}
$$

\section{e.2) Situation Involving the Initial Distance $I_{0}$ in Space, with 11 Initial} Paths (from Case a.2)

We now recalculate the magnitude of the force of interaction $F_{P}$ between MP1 and MP2, by considering all spatial paths for the initial distance $l_{0}$, starting from the six paths in Section 6.2. The number of these paths will be in accordance with the rule above:

$$
N^{\text {tot }}=1+6+13+19+25+31+37+43+49+55+61=340 \text { pcs }
$$

The magnitude of the $F_{p}$ interaction force is obtained from (24a) using (49):

$$
\begin{aligned}
F_{P}^{\text {tot }}= & \frac{k_{p}}{\left(l_{0}\right)^{2}}[1 \times 1+0.9227 \times 6+0.8541 \times 13+0.793 \times 19+0.738 \times 25 \\
& +0.6886 \times 31+0.644 \times 37+0.6037 \times 43 \\
& +0.567 \times 49+0.5335 \times 55+0.503 \times 61] \\
= & \frac{k_{p}}{\left(l_{0}\right)^{2}}[1+5.536+11.10+15.06+18.45+21.34 \\
& +23.83+25.96+27.78+29.34+30.68] \\
= & \frac{k_{p}}{l_{0}^{2}} \times 210.10
\end{aligned}
$$

\section{f.1) Situation Involving the Doubled Distance $\left(2 I_{0}\right)$ in Space, with 6 Initial}

\section{Paths (from Case b.1)}

We now recalculate the magnitude of the force of interaction $F_{P}$ between MP1 and MP2, by considering all spatial paths for the doubled initial distance $2 I_{0}$, starting from the 6 paths in Section 6.2. The number of these paths will be in accordance with the rule above:

$$
N^{\text {tot }}=1+6+13+19+25+31=95 \text { pcs }
$$

The magnitude of the interaction force $F_{P}$ is obtained from (27a) using (51):

$$
\begin{aligned}
F_{P}^{\text {tot }}= & \frac{k_{p}}{\left(2 l_{0}\right)^{2}}[1 \times 1+0.854 \times 6+0.738 \times 13+0.644 \times 19 \\
& +0.567 \times 25+0.503 \times 31] \\
= & \frac{k_{p}}{\left(2 l_{0}\right)^{2}}[1+5.12+9.594+12.23+14.17+15.59] \\
= & \frac{k_{p}}{\left(2 l_{0}\right)^{2}} \times 57.704=\frac{k_{p}}{l_{0}^{2}} \frac{1}{4} \times 57.704=\frac{k_{p}}{l_{0}^{2}} \times 14.42
\end{aligned}
$$


f.2) Situation Involving the Doubled Distance $\left(2 I_{0}\right)$ in Space, with 11 Initial Paths (from Case b.2)

We now recalculate the magnitude of the force of interaction $F_{P}$ between MP1 and MP2, by considering all spatial paths for the doubled initial distance $21_{0}$, starting from the 11 paths in Section 6.2. The number of these paths will be in accordance with the rule above:

$$
N^{\text {tot }}=1+6+13+19+25+31+37+43+49+55+61=340 \text { pcs }
$$

The magnitude of the interaction force $F_{P}$ is obtained from (29a) using (53):

$$
\begin{aligned}
F_{P}^{\text {tot }}= & \frac{k_{p}}{\left(2 l_{0}\right)^{2}}[1 \times 1+0.9227 \times 6+0.8541 \times 13+0.793 \times 19+0.738 \times 25 \\
& +0.6886 \times 31+0.644 \times 37+0.6037 \times 43 \\
& +0.567 \times 49+0.5335 \times 55+0.503 \times 61] \\
= & \frac{k_{p}}{\left(2 l_{0}\right)^{2}}[1+5.536+11.10+15.06+18.45+21.34 \\
& +23.83+25.96+27.78+29.34+30.68] \\
= & \frac{k_{p}}{l_{0}^{2}} \frac{1}{4} \times 210.10=\frac{k_{p}}{l_{0}^{2}} \times 52.52
\end{aligned}
$$

f.3) Situation Involving the Doubled Distance $\left(2 I_{0}\right)$ in Space, with 21 Initial Paths (from Case b.3)

We now recalculate the magnitude of the force of interaction $F_{P}$ between MP1 and MP2, by considering all spatial paths for the doubled initial distance $21_{0}$, starting from the 21 paths in Section 6.2. The number of these paths will be in accordance with the rule above:

$$
\begin{aligned}
N^{\text {tot }}= & 1+6+13+19+25+31+37+43+49+55+61+67 \\
& +73+79+85+91+97+103+109+115+121 \\
= & 340+940=1290 \mathrm{pcs}
\end{aligned}
$$

The magnitude of the $F_{P}$ interaction force is obtained from (31a) using (55):

$$
\begin{aligned}
F_{P}^{\text {tot }}= & \frac{k_{p}}{\left(2 l_{0}\right)^{2}}[1+0.960 \times 6+0.9227 \times 13+0.887 \times 19+0.8541 \times 25 \\
& +0.8227 \times 31+0.793 \times 37+0.7647 \times 43+0.738 \times 49 \\
& +0.7126 \times 55+0.6886 \times 61+0.6658 \times 67+0.644 \times 73 \\
& +0.6234 \times 79+0.6037 \times 85+0.5849 \times 91+0.567 \times 97 \\
& +0.5499 \times 103+0.5335 \times 109+0.5179 \times 115+0.503 \times 121] \\
= & \frac{k_{p}}{\left(2 l_{0}\right)^{2}}[1+5.76+11.995+16.853+21.35+25.50+29.34 \\
+ & 32.88+36.16+39.19+42.0+44.61+47.0+49.25 \\
+ & 51.31+53.22+54.99+56.64+58.15+59.56+60.86] \\
= & \frac{k_{p}}{\left(2 l_{0}\right)^{2}}(1+796.62)=\frac{k_{p}}{\left(l_{0}\right)^{2}} \frac{1}{4} \times 797.62=\frac{k_{p}}{\left(l_{0}\right)^{2}} \times 199.40
\end{aligned}
$$




\subsection{Comparison of the Results from the Previous Situations from Sections 6.2.1 and 6.3, with Conclusions}

In Sections 6.2.1 and 6.3, based on the analysis of the variation with distance $I$ of the force of electrical interaction $F_{P}$ between the two MPs (See Section 6.1.), a series of numerical calculations was performed for some particular cases involving the distance $I_{0}$ between MP1 and MP2, the limit of angle $\alpha$, the spatial distribution of paths and the path deformation $\varepsilon$. The initial conditions and results of calculations for the forces $F_{P}$ are presented in Table 1.

The purpose of this calculation was to establish a new correlation between the magnitude of the force $F_{P}$ and the change in the distance $I$ between MP1 and MP2. For the distance between MP1 and MP2, multiple integer values of an initial distance $I_{0}$, i.e. $l_{0}, 2 l_{0}, 3 l_{0}, \cdots$ were considered.

However, it was sufficient for generalization purposes to use only the first two multiples of the distance $I_{0}$, i.e., $I_{0}$ and $2 l_{0}$. For $n$ paths $I_{i}$ between MP1 and MP2, 6,11 and 21 paths were considered, which proved to be sufficiently precise for generalization.

Table 1. Results of calculations for forces $F_{P}$ from all above Cases.

\begin{tabular}{|c|c|c|c|c|c|c|c|}
\hline Cases/Paragr. & Anga $\left({ }^{\circ}\right)$ & Plan/ zone & Dist. & No. $n+1$ & $\begin{array}{l}\text { Deformation } \\
\qquad \varepsilon\end{array}$ & $\begin{array}{l}\text { Force } \\
\qquad F_{P}\end{array}$ & $\begin{array}{c}\text { Final } \\
F_{P}\end{array}$ \\
\hline a.1) & 45 & up & $l_{0}$ & 6 & $\varepsilon=\Delta l / l_{0}=0.082$ & $(1+3.35) \cdot k_{p} / l_{0}^{2}$ & $4.35 \cdot k_{p} / l_{0}^{2}$ \\
\hline a.1).s & 45 & up & $I_{0}$ & 9 & $\varepsilon=\Delta l / l_{0}=0.05125$ & $(1+5.432) \cdot k_{p} / l_{0}^{2}$ & $6.432 \cdot k_{p} / l_{0}^{2}$ \\
\hline a.2) & 45 & up & $l_{0}$ & 11 & $\varepsilon=\Delta l / l_{0}=0.041$ & $(1+6.847) \cdot k_{p} / l_{0}^{2}$ & $7.847 \cdot k_{p} / l_{0}^{2}$ \\
\hline b.1) & 45 & up & $2 I_{0}$ & 6 & $\varepsilon=\Delta l / 2 l_{0}=0.082$ & $(1+3.306) \cdot k_{p} /\left(2 l_{0}^{2}\right)$ & $1.0765 \cdot k_{p} / l_{0}^{2}$ \\
\hline b.2) & 45 & up & $2 I_{0}$ & 11 & $\varepsilon=\Delta l / 2 l_{0}=0.041$ & $(1+6.847) \cdot k_{p} /\left(2 l_{0}^{2}\right)$ & $1.962 \cdot k_{p} / l_{0}^{2}$ \\
\hline b.2).s & 45 & up & $2 I_{0}$ & 17 & $\varepsilon=\Delta l / 2 l_{0}=0.02562$ & $(1+11.10) \cdot k_{p} /\left(2 l_{0}^{2}\right)$ & $3.045 \cdot k_{p} / l_{0}^{2}$ \\
\hline b.3) & 45 & up & $2 I_{0}$ & 21 & $\varepsilon=\Delta l / 2 l_{0}=0.0205$ & $(1+13.271) \cdot k_{p} /\left(2 l_{0}^{2}\right)$ & $3.318 \cdot k_{p} / l_{0}^{2}$ \\
\hline c.1)/a.1) & 45 & $\mathrm{u} / \mathrm{d}$ & $l_{0}$ & 6 & $\varepsilon=\Delta l / l_{0}=0.082$ & $(1+6.70) \cdot k_{p} / l_{0}^{2}$ & $7.70 \cdot k_{p} / l_{0}^{2}$ \\
\hline /a.2) & 45 & $\mathrm{u} / \mathrm{d}$ & $I_{0}$ & 11 & $\varepsilon=\Delta l / l_{0}=0.041$ & $(1+13.694) \cdot k_{p} / l_{0}^{2}$ & $14.694 \cdot k_{p} / l_{0}^{2}$ \\
\hline$/ \mathrm{b} .1)$ & 45 & $\mathrm{u} / \mathrm{d}$ & $2 I_{0}$ & 6 & $\varepsilon=\Delta l / 2 l_{0}=0.082$ & $(1+6.612) \cdot k_{p} /\left(2 l_{0}^{2}\right)$ & $1.934 \cdot k_{p} / l_{0}^{2}$ \\
\hline$/ \mathrm{b} .2)$ & 45 & $\mathrm{u} / \mathrm{d}$ & $2 I_{0}$ & 11 & $\varepsilon=\Delta l / 2 l_{0}=0.041$ & $(1+13.694) \cdot k_{p} /\left(2 l_{0}^{2}\right)$ & $3.673 \cdot k_{p} / l_{0}^{2}$ \\
\hline /b.3) & 45 & $\mathrm{u} / \mathrm{d}$ & $2 I_{0}$ & 21 & $\varepsilon=\Delta l / 2 l_{0}=0.0205$ & $(1+26.542) \cdot k_{p} /\left(2 l_{0}^{2}\right)$ & $6.885 \cdot k_{p} / l_{0}^{2}$ \\
\hline d.1) & 60 & up & $l_{0}$ & 10 & $\varepsilon=\Delta l / l_{0}=0.111$ & $(1+4.138) \cdot k_{p} / l_{0}^{2}$ & $5.138 \cdot k_{p} / l_{0}^{2}$ \\
\hline d.2) & 60 & up & $2 I_{0}$ & 19 & $\varepsilon=\Delta l / 2 l_{0}=0.055$ & $(1+8.8696) \cdot k_{p} /\left(2 l_{0}^{2}\right)$ & $2.467 \cdot k_{p} / l_{0}^{2}$ \\
\hline e.1) & 45 & cone & $I_{0}$ & 6 & $\varepsilon=\Delta l / l_{0}=0.082$ & $(1+57.29) \cdot k_{p} / l_{0}^{2}$ & $58.29 \cdot k_{p} / l_{0}^{2}$ \\
\hline e.2) & 45 & cone & $I_{0}$ & 11 & $\varepsilon=\Delta l / l_{0}=0.041$ & $(1+209.10) \cdot k_{p} / l_{0}^{2}$ & $210.10 \cdot k_{p} / l_{0}^{2}$ \\
\hline f.1) & 45 & cone & $2 I_{0}$ & 6 & $\varepsilon=\Delta l / 2 l_{0}=0.082$ & $(1+56.704) \cdot k_{p} /\left(2 l_{0}^{2}\right)$ & $14.42 \cdot k_{p} / l_{0}^{2}$ \\
\hline f.2) & 45 & cone & $2 I_{0}$ & 11 & $\varepsilon=\Delta l / 2 l_{0}=0.041$ & $(1+209.10) \cdot k_{p} /\left(2 l_{0}^{2}\right)$ & $52.52 \cdot k_{p} / l_{0}^{2}$ \\
\hline f.3) & 45 & cone & $2 I_{0}$ & 21 & $\varepsilon=\Delta l / 2 l_{0}=0.0205$ & $(1+796.60) \cdot k_{p} /\left(2 l_{0}^{2}\right)$ & $199.1 \cdot k_{p} / l_{0}^{2}$ \\
\hline
\end{tabular}


It was intended to obtain information on the $F_{P}$ force variation when changing both the above parameters: the distance $l$ and the number of paths $n+1$, where $n$ represents the side paths, but not the central path.

However, in order to have comparable values for the two values of the $F_{P}$ force, when both of the above parameters (number $n$ of paths and distance $l_{0}$ ) change, we firstly compare the situation for the distance $2 I_{0}$ with that for the distance $I_{0}$ (Figure 7, Figure 9).

A). For the plane situation, to highlight the effect of doubling the distance $l_{0} \rightarrow 21_{0}$ on $F_{P}$ the following 4 combinations of cases a.) to f.) from Table 1 are considered and compared, analysing the forces ratio $r_{F}=F_{P 2} / F_{P 1}$ for $2 l_{0}$ and for $l_{0}$ at corresponding paths number $n+1$, of 6,11 and 21 .

i). The plane situation with upward paths (u) with $11 / 6$ paths, Case b.2) with $F_{P 2}$, versus Case a.1) with $F_{P 1}$, give the next values for the forces $F_{P}$ ratio $r_{F}$.

$$
r_{F}=F_{P 2} / F_{P 1}=1.962 / 4.35=0.45
$$

i).s. The plane situation with upward paths (u) with 17/9 paths, Case b.2).s. with $F_{P 2}$, versus Case a.1).s. with $F_{P 1}$, give the next values for the forces $F_{P}$.

$$
r_{F}=F_{P 2} / F_{P 1}=3.045 / 6.432=0.473
$$

One remark here that increasing the paths number $n+1$, from 6 to 9 , gives only a little influence on $r_{F}$, so the number of 6 paths utilised above is correct and sufficient.

ii). The plane situation with upward paths (u) with 21/11 paths, Case b.3) with $F_{P 2}$, versus Case a.2) with $F_{P 1}$, give the next values for the forces $F_{P}$.

$$
r_{F}=F_{P 2} / F_{P 1}=3.318 / 7.847=0.42
$$

iii). The plane situation as in c.1) with upward and downward paths $(\mathrm{u} / \mathrm{d})$ with $11 / 6$ paths, Case c.1)/b.2) with $F_{P 2}$, versus Case c.1)/a.1) with $F_{P 1}$, give the next values for the forces $F_{P}$.

$$
r_{F}=F_{P 2} / F_{P 1}=3.67 / 7.70=0.47
$$

iv). The plane situation as in Case c.1) with upward and downward paths (u/d) with $21 / 11$ paths Case c.1)/b.3) with $F_{P 2}$, versus Case c.1)/a.2) with $F_{P 1}$, give the next values for the forces $F_{P}$.

$$
r_{F}=F_{P 2} / F_{P 1}=6.8857 / 14.694=0.47
$$

The plane situation for the paths results in a decrease in $F_{P 2}$ of approximately $54.4 \%$ (mean ratio $r_{F}=0.456$ ) of $F_{P 1}$, by doubling the distance $/$ between MPs.

However, the decrease in $F_{C}$ force ratio $r_{F}=$ according to Coulomb's law for doubling the distance 1 , is:

$$
r_{F}=F_{C 2} / F_{C 1}=\left[1 /\left(2 l_{0}\right)^{2}\right] /\left[1 /\left(l_{0}\right)^{2}\right]=1 / 4=0.25 \ll 0.456
$$

This indicates a decrease in $F_{P}$ force with only $54.7 \%$ compared with $75.0 \%$ decrease for $F_{C}$ force. Even in this case, with only incomplete plane paths, result of Coulomb's law differs markedly from Equations (57)-(60).

B). For the spatial situation with a conic at $2 \times 45^{\circ}$ distribution of paths, to 
highlight the effect of doubling the distance $I_{0} \rightarrow 21_{0}$ on $F_{P}$ the following two Cases from Table 1 are considered and compared.

i). The spatial situation, with $11 / 6$ paths, Case f.2) with $F_{P 2}$, versus Case e.1) with $F_{P 1}$, give the next values for the forces $F_{P}$ ratio $r_{F}$

$$
r_{F}=F_{P 2} / F_{P 1}=52.52 / 58.29=0.90
$$

ii). The spatial situation, with $21 / 11$ paths, Case f.3) with $F_{P 2}$, versus Case e.2) with $F_{P 1}$, give the next values for the forces $F_{P \text {. }}$

$$
r_{F}=F_{P 2} / F_{P 1}=199.0 / 210.1=0.947
$$

One remark here that the spatial situation from $B$ ), compared with the plane situation from A) gives an important favourable influence on $r_{F}$ ratio, which has increased strongly, so only the spatial situation must be considered in future, which is also physically justified.

The spatial situation of paths from ii). gives an $r_{F}$ ratio of $F_{P}$ forces from relation (63) which indicate a diminution of $F_{P}$ forces with about 5.3\% (ratio 0.947), by doubling the distance between MPs.

This also must be compared with the $r_{F}$ ratio of $F_{C}$ forces according to Coulomb's law, where doubling the distance $l_{0}$ from (61), results in:

$$
r_{F}=F_{C 2} / F_{C 1}=0.25 \ll 0.947
$$

Also, in this spatial case, Coulomb's law in Equation (61) is more clearly not confirmed by result from (63).

This indicates a decrease in $F_{p}$ force with only $5.3 \%$ compared with the decrease of $75.0 \%$ (ratio 0.25 ) for $F_{C}$ force by doubling distance $I_{0}$.

It appears probable, that by increasing the number $n$ of paths beyond $n=21$, one could obtain a ratio $r_{F}=F_{P 2} / F_{P 1}$ greater than 0.947 or equal to 1.0 , or even greater than 1.0.

However, the result from (63) provides strong support for justifying the introduction of the term $\ln r$ in the $F_{C C}$ formula from [3].

This justification appears because, in this case of $F_{C O}$ the effect of doubling the distance from $I_{0}$ to $2 I_{0}$ is the next:

$$
\begin{aligned}
r_{F} & =F_{C C 2} / F_{C C 1}=\ln \left(2 l_{0}\right)^{2} / \ln l_{0}^{2}=2 \cdot \ln \left(2 l_{0}\right) / 2 \cdot \ln l_{0} \\
& =\ln 2 l_{0} / \ln l_{0}=\ln 2 / \ln l_{0}+1
\end{aligned}
$$

We observe in (65) that for long distances $I_{0}$, the first term becomes zero and the ratio $r_{F}$ of $F_{C C}$ forces tends to 1.0 .

Consequently, the result from (63) is almost the same as that from (65) for long distances, and hence this result justifies the presence of the term $\ln r$ in the formula for the $F_{C C}$ force from [1].

We remark that in any of the pairs of comparable cases above from A) and B), the condition of the equality of maximum intervals $\Delta d_{2}=\Delta d_{1}$ was considered, between two successive paths, at the midpoint of the distance between the two MPs (Figure 8). This is the physical justification of the equivalence and comparability of any pair of paths as above from A) and B). 
We observe that the result obtained in (63) for the electrical interaction force $F_{P}$ between MP2 and MP1, indicates a spatial effect of the ether which leads at a limit of $r_{F}$ to 1.0 in the decrease of the $F_{P}$ forces ratio when doubling the distance l, in contrast to Coulomb's $F_{C}$ forces whose ratio $r_{F}$ decreases to 0.25 with doubling 1 .

But this result for $F_{P}$ is in concordance with the presence of term $\ln r$ in formula of $F_{C C}$ force from [1].

We must remark that results for all the Cases above analysed, when we doubled the distances from $I_{0}$ to $2 I_{0}$, can be generalised for new doubled distances: $2 l_{0}, 4 l_{0}, 8 l_{0}, 16 l_{0}, 32 l_{0}, \cdots$, when the first forces $F$ ratio $r_{F}$ must be put at the correspondent power:

$$
r_{F},\left(r_{F}\right)^{2},\left(r_{F}\right)^{3},\left(r_{F}\right)^{4},\left(r_{F}\right)^{5}, \cdots
$$

In case of $F_{C}$ force, the first ratio $r_{F}=0.25$ put at rising powers from (66) will tend quickly to zero.

But in case of $F_{P}$ force, the first ratio $r_{F}=0.95, \cdots, 1.0$, put at rising powers from (66) will tend to approx. 1.0, as tends also the $r_{F}$ ratio for term $\ln r$, (in 65), so being justified its presence.

The physical explanation for this result from (63) lies in the presence around MP1 and MP2 of ether cells (ECs), which transmit the percussion forces $F_{P}$ not by a single direct path but by multiple paths between MP1 and MP2, spatially distributed, but some of them simultaneously arriving at MP2, so that their effect is superposed.

These multiple forces $F_{P}$ continue to arrive at MP2 at any moment in time, and hence they accumulate. The number $n$ of paths $l_{i}$ increases as the distance is doubled, compensating for the diminution of the percussion forces $F_{P}$ by $1 / \mathbb{R}$ term.

\section{Conclusions and Consequences}

In this paper, we analysed the role of the ether and the effect of its presence in nature, in the form of our HM16 model, with reference to the interaction between two MPs situated at the distance 1 . The interaction consists of forces $F_{P}$, produced by mechanical percussions between MPs through the ether vibrations FVs, and depending on $1 / P$. But the forces $F_{P}$ constitute in fact the so called electric Coulomb's forces $F_{O}$ between so called electric charges, which charges in fact do not exist as a physical quantity.

We have shown that the force $F_{p}$, regarded as the basic elementary electric force, can be considered as the resultant force of multiple percussions $p_{i}$ on an MP. These percussions are produced by the vibrating ECs of the ether and are transmitted by the fundamental vibrations (FVs) created in ether by other MPs. Was calculated that individual $F_{P}$ force given by the ether percussions at astronomical distances is practically equal with $F_{O}$ but at laboratory distance discrepancies appear, indicating the theory applicability at long distances. 
An initial assessment of the all $F_{P}$ forces upon an MP was made in the paper, using a simplified numerical calculation, aimed to determine the effect of all FVs travelling along various rays/paths $l_{i}$ through the ether, between two MPs considered today as having electric charges.

However, a series of such paths departing in a given time interval from MP1, at different angles from the direct path to MP2, but inside an angle of $45^{\circ}$, will arrive at the same moment $t_{0}$ at MP2, with the result that their individual forces $F_{P i}$ cumulates their effects, and so compensating the decrease of $F_{P}$ due to the term in $1 / P$.

As a result of these multiple paths and of associated forces $F_{P \text { p }}$ a limited to 1.0 decrease in the final $F_{P}$ force was obtained in the calculations, along with an increase in the distance 1 , in contrast to Coulomb's law, where $F_{C}$ decreases to zero with increasing $l$, due to the term in $1 / P$.

The effects of these multiple paths, of associated forces $F_{P,}$ and of the spatial presence of the ether, were evaluated by numerical calculus which furnished a limit to 1.0 in the decrease of the $F_{P}$ forces ratio, when doubling the distance 1. This result is in contrast to Coulomb's $F_{C}$ forces, whose ratio decreases to 0.25 with doubling 1 , and to zero after multiples doublings.

Accordingly, the possibility of including the term $\ln r$ in the $F_{C C}$ forces was demonstrated, because the ratio of decreasing of $\ln r$ is capped also to 1.0 for doubling $l$ at long distances.

These results justify the possibility and necessity of introducing the term $\ln r$ into the $F_{C C}$ force in Equations (21) in [3], which will be maintained in our future analyses.

As a consequence of the above results validating the $F_{C C}$ force formula, the electrical nature of gravity based on the electrical dipole constitution of matter, is confirmed, since numerical results given by $F_{D C}$ force as gravity force, are very close to those gave by Newton's law force $F_{N}[3]$.

Our "new gravity" theory, starting from the $F_{C C}$ electric force in [3], involves a more complex force law of gravity, considering for this the $F_{D C}$ force between the electrical dipoles of all matter. The formula of $F_{D C}$ force is constituted by a series of powers of $r^{x}$, which is different from Newton's simple gravitational force $F_{N}$.

However, the new form of the gravitational force $F_{D C}$ will explain special phenomena observed elsewhere.

The above results through $F_{D C}$ as gravitation force, undermine the usefulness of gravity wave searches, of black hole hunting and of searches for dark matter and dark energy, which can all (among other things) be explained by the new gravity force $F_{D C}$.

Obviously, our overall analysis of gravity started earlier in 2000, with the admission of the existing error in Michelson's calculation [9], resulting immediately in the nonutility of Special Relativity Theory (SRT), along with the readmission of the ether into physics, according to the HM16 model. The ether presence indicates the uselessness of General Relativity (GR), and of Newton's 
law force $F_{N}$, which can be replaced by the $F_{D C}$ force, appearing between any two electrical dipoles constituents of all the matter, at any distance. But the $F_{D C}$ force complex general equation, needs to be developed and applied to different practical situations, by physics community.

\section{Acknowledgements}

The first author acknowledges the initial advice on the subject and the encouragement of his late professor N. Barbulescu. He is sincerely grateful to the late Prof. P. Mazilu from TUCB Bucharest for his rigorous lessons on rationality. $\mathrm{He}$ is also indebted to Gen. Prof. G. Barsan, Col. Prof. Al. Babos from LFA Sibiu, Prof. D. Stoicescu from ULBS Sibiu, Prof. D. Siposan from MTA Bucharest and Miss Veronica Has from Bucharest, for their continued or past support. For the first developments of this theme, the authors appreciate the support of the MEC (grant under contract no. 05-D11-54/2005).

\section{Conflicts of Interest}

The authors declare no conflicts of interest regarding the publication of this paper.

\section{References}

[1] Has, I. and Miclaus, S. (2017) Physics Essays, 30, 45-56. https://doi.org/10.4006/0836-1398-30.1.45

[2] Has, I., Miclaus, S. and Has, A. (2018) Journal of Applied Mathematics and Physics, 6, 1886-1895. https://doi.org/10.4236/jamp.2018.69160

[3] Has, I., Miclaus, S. and Has, A. (2015) American Journal of Modern Physics, 4, 97-108. https://doi.org/10.11648/j.ajmp.20150403.11

[4] Whittaker, E.T. (1910) A History of the Theories of Aether and Electricity. Longman Green and Co., London; Hodges, Figgis and Co., Dublin.

[5] Feynman, R.P. (1964) Lectures in Physics. Vol. 2: Mainly Electromagnetism and Matter. Addison-Wesley, Reading.

[6] Purcell, E.M. (1982) Electricity and Magnetism. Berkley Physics Course Vol. 2, Editura Didactica \& Pedagogica, Bucharest. (In Romanian)

[7] Barbulescu, N. (1962) Elements of General Physics. Editura Didactica \& Pedagogica, Bucharest. (In Romanian)

[8] Luca, E., Ciubotariu, C., Maftei, G., Zet, G., Jeflea, A. and Pasnicu, C. (1996) Physics. Vol. 2, Editura Stiintifica, Bucharest. (In Romanian)

[9] Has, I., Miclaus, S. and Has, A. (2018) Journal of Applied Mathematics and Physics, 6, 1507-1521. https://doi.org/10.4236/jamp.2018.67127 